\title{
NUMERICAL APPROXIMATION OF THE BOUNDARY CONTROL FOR THE WAVE EQUATION IN A SQUARE WITH MIXED FINITE ELEMENTS
}

\author{
Carlos Castro $\quad$ Sorin Micu Arnaud Münch
}

\begin{abstract}
This paper studies the numerical approximation of the boundary control for the wave equation in a square domain. It is known that the discrete and semi-discrete models obtained by discretizing the wave equation with the usual finite difference or finite element methods do not provide convergent sequences of approximations to the boundary control of the continuous wave equation, as the mesh size goes to zero (see $[7,15]$ ). Here we introduce and analyze a new semi-discrete model based on the space discretization of the wave equation using a mixed finite element method with two different basis functions for the position and velocity. The main theoretical result is a uniform observability inequality which allows us to construct a sequence of approximations converging to the minimal $L^{2}$-norm control of the continuous wave equation. We also introduce a fully-discrete system, obtained from our semi-discrete scheme, for which we conjecture that it provides a convergent sequence of discrete approximations as both $h$ and $\Delta t$, the time discretization parameter, go to zero. We illustrate this fact with several numerical experiments.
\end{abstract}

ETSI de Caminos, Canales y Puertos, Universidad Politéenica de Madrid, 28040 Madrid, Spain (ccastro@caminos. upm.es). Partially supported by Grant BFM 2002-03345 of MCYT (Spain).

Facultatea de Matematica-Informatica, Universitatea din Craiova, Romania (sd_nicu@yahoo.com). Partially supported by Grants MTM2005-00714 of MCYT (Spain), CNCSIS 80/2005 and CEEX-05-D11-36/2005 (Romania).

Laboratoire de Mathématiques de Besançon, UMR CNRS 6623, Université de Franche-Comte, 16 route de Gray, 25030 Besançon cedex, France (arnaud.munchomath.univ-fcomte.fr). Partially supported by the EU Grant HPRN-CT-2002-00284 New materials, adaptive systems and their nonlinearities: modelling, control and numerical simulation 


\section{Introduction}

Let us consider $\Omega=(0,1) \times(0,1) \subset \mathbb{R}^{2}$ with boundary $\Gamma=\Gamma_{0} \cup \Gamma_{1}$ divided as follows

$$
\left\{\begin{array}{l}
\Gamma_{0}=\{(x, 0): 0 \leq x \leq 1\} \cup\{(0, y): 0 \leq y \leq 1\} \\
\Gamma_{1}=\{(x, 1): 0<x<1\} \cup\{(1, y): 0<y<1\} .
\end{array}\right.
$$

We are concerned with the following exact boundary controllability property for the wave equation in $\Omega$ : given $T$ sufficiently large and $\left(u^{0}, u^{1}\right) \in L^{2}(\Omega) \times H^{-1}(\Omega)$ there exists a control function $(v(t, y), z(t, x)) \in\left[L^{2}((0, T) \times(0,1))\right]^{2}$ such that the solution of the equation

$$
\begin{cases}u^{\prime \prime}-\Delta u=0 & \text { for }(x, y) \in \Omega, t>0 \\ u(t, x, y)=0 & \text { for }(x, y) \in \Gamma_{0}, t>0 \\ u(t, 1, y)=v(t, y) & \text { for } y \in(0,1), t>0 \\ u(t, x, 1)=z(t, x) & \text { for } x \in(0,1), t>0 \\ u(0, x, y)=u^{0}(x, y) & \text { for }(x, y) \in \Omega \\ u^{\prime}(0, x, y)=u^{1}(x, y) & \text { for }(x, y) \in \Omega\end{cases}
$$

satisfies

$$
u(T, \cdot)=u^{\prime}(T, \cdot)=0
$$

By' we denote the time derivative.

The Hilbert Uniqueness Method (HUM) introduced by J.-L. Lions provides a control $(v, z)$ with minimal $L^{2}$-norm . This control is unique and it will be referred in the sequel as the HUM control. We briefly describe this method at the beginning of section 2 below.

In the last years many works have dealt with the numerical approximations of the control problem (1.2)-(1.3). For instance, , numerical algorithms based on the finite difference and finite element approximations of (1.2) were described. However, these algorithms do not converge when the discretization parameters go to zero.

Let us briefly explain this fact. When we are dealing with the exact controllability problem, a uniform time $T>0$ for the control of all solutions is required. This time $T$ depends on the size of the domain and the velocity of propagation of waves. In general, any semi-discrete dynamics generates spurious high-frequency oscillations that do not exist at the continuous level. Moreover, a numerical dispersion phenomenon appears and the velocity of propagation of some of these high frequency numerical waves may possibly converge to zero when the mesh size $h$ 
does. Consequently, the controllability property for the semi-discrete system will not be uniform for a fixed time $T$. This is the case when the semi-discrete model is obtained by discretizing the wave equation with the usual finite-difference or finite element method (see for a detailed analysis of the 1-D case and for the 2-D case, in the context of the dual observability problem).

From the numerical point of view, several techniques have been proposed as possible cures of the low velocity of propagation of the high frequency spurious oscillations

. To our knowledge, no proof of convergence has been given for any of these methods, as $h$ tends to 0, so far.

In this paper we construct, for any $T$ sufficiently large but independent of $h$, a convergent sequence of semi-discrete approximations of the HUM control $(v, z)$ of $(1.2)$. The main idea is to introduce a new space discretization scheme for the wave equation (1.2), based on a mixed finite element method, in which different base functions for the position $u$ and the velocity $u^{\prime}$ are considered. More precisely, while the usual linear finite elements are used for the former, discontinuous elements approximate the latter. This new scheme still has spurious high-frequency oscillations but the numerical dispersion makes them to have larger velocity of propagation. Consequently, the velocity of propagation of all waves is bounded from below by a uniform positive constant.

The semi-discrete approximations $\left(v_{h}, z_{h}\right)_{h>0}$ of the HUM control $(v, z)$ of $(1.2)$ are obtained by minimizing a functional $\mathcal{J}_{h}$ depending on the associated space discretized adjoint system (see (5.1)). The main result of the paper is Theorem 4.1 which gives a uniform (in $h$ ) observability inequality for this homogeneous semi-discrete adjoint system. This is equivalent to the uniform coercivity of $\mathcal{J}_{h}$. Theorem 4.1 permits to show that, if a weakly convergent sequence of approximations of the continuous initial data $\left(u^{0}, u^{1}\right)$ is considered, the sequence of approximations $\left(v_{h}, z_{h}\right)_{h>0}$ converges weakly to $(v, z)$ (Theorems 6.2 and 6.3 ).

To our knowledge, the scheme described in this paper was used by the first time in the context of the wave equation in [1], in order to obtain a uniform decay rate of the energy associated to the semi-discrete wave equation by a boundary dissipation. This scheme is different from the mixed element method applied in [5] where two different basis functions are considered for $u$ and $\nabla u$.

In this paper, we concentrate on the simplest 2-D domain consisting of a unit square. The 
mixed finite element method may be applied to general domains but our proofs of the uniform observability and convergence strongly depend on the particular geometry of the square and cannot be generalized.

We also introduce a fully-discrete approximation of the wave equation, based on the semidiscrete scheme, for which the velocity of propagation of all numerical waves does not vanish as both $h$ and $\Delta t$, the time discretization parameter, tend to zero. Based on this fact, we conjecture that this fully-discrete scheme also provides convergent approximations of the control. At the end, we include two numerical experiments that illustrate this fact.

The rest of the paper is organized in the following way. The second section briefly recalls some controllability results for the wave equation (1.2) and introduces the Hilbert Uniqueness Method. In the third section the semi-discrete model under consideration is deduced. In the fourth section the main properties of this system are discussed and, in particular, the fundamental uniform observability inequality (Theorem 4.1). Its technical proof is given in an Appendix at the end of the paper. In the fifth section an approximation sequence is constructed and in the sixth section its convergence to the HUM control of the continuous equation (1.2) is proved. The final section is devoted to present the fully-discrete scheme and the numerical results.

\section{The continuous problem: results and notations}

In this section we recall some of the controllability properties of the wave equation (1.2) and we briefly describe the Hilbert Uniqueness Method. Also, we introduce some notations that will be used in the article. The following classical result may be found,

Theorem 2.1 Given any $T>2 \sqrt{2}$ and $\left(u^{0}, u^{1}\right) \in L^{2}(\Omega) \times H^{-1}(\Omega)$ there exists a control function $(v, z) \in\left[L^{2}((0, T) \times(0,1))\right]^{2}$ such that the solution $\left(u, u^{\prime}\right)$ of (1.2) verifies (1.3).

In general, there are infinitely many controls, when they exist. However, the one with minimal $L^{2}$-norm is unique and can be characterized by the minimizer of a suitable functional. Let us introduce the map $\mathcal{J}: H_{0}^{1}(\Omega) \times L^{2}(\Omega) \rightarrow \mathbb{R}$ defined by

$$
\begin{gathered}
\mathcal{J}\left(w^{0}, w^{1}\right)=\frac{1}{2} \int_{0}^{T} \int_{0}^{1}\left(w_{x}\right)^{2}(t, 1, y) d y d t+\frac{1}{2} \int_{0}^{T} \int_{0}^{1}\left(u_{y}\right)^{2}(t, x, 1) d x d t \\
+\int_{\Omega} u^{0}(x, y) w^{\prime}(0, x, y) d x d y-\left\langle u^{1}, w(0, \cdot)\right\rangle_{-1,1}
\end{gathered}
$$


where $\left(u, w^{\prime}\right)$ is the solution of the backward homogeneous equation

$$
\left\{\begin{array}{lr}
w^{\prime \prime}-\Delta w=0, & \text { for }(x, y) \in \Omega, t>0, \\
w(t, 0, y)=w(t, x, 0)=w(t, x, 1)=w(t, 1, y)=0, & \text { for } x, y \in(0,1), t>0, \\
w(T, x, y)=w^{0}(x, y), w^{\prime}(T, x, y)=w^{1}(x, y), & \text { for }(x, y) \in \Omega .
\end{array}\right.
$$

In $(2.1),<\cdot, \cdot>_{-1,1}$ denotes the duality product between $H^{-1}(\Omega)$ and $H_{0}^{1}(\Omega)$.

Theorem 2.2 Given any $T>2 \sqrt{2}$ and $\left(u^{0}, u^{1}\right) \in L^{2}(\Omega) \times H^{-1}(\Omega), \mathcal{J}$ has an unique minimizer $\left(\widehat{w}^{0}, \widehat{w}^{1}\right) \in H_{0}^{1}(\Omega) \times L^{2}(\Omega)$. If $\left(\widehat{w}, \widehat{w}^{\prime}\right)$ is the solution of (2.2) with initial data $\left(\widehat{w}^{0}, \widehat{w}^{1}\right)$, then

$$
(v(t, y), \tilde{z}(t, x))=\left(\widehat{w}_{x}(t, 1, y), \widehat{w}_{y}(t, x, 1)\right)
$$

is the control of (1.2) with minimal $L^{2}-$ norm.

The method we have just presented was introduced by J.-L. Lions (see [10]) and named Hilbert Uniqueness Method (HUM). The control $(v, z)$ given by (2.3) is usually called the HUM control.

We recall that the main ingredient of the proof of the Theorem 2.2 is the following observability inequality for $(2.2)$ : given $T>2 \sqrt{2}$ there exists a constant $C>0$ such that the following inequality holds for any solution of $(2.2)$,

$$
\int_{\Omega}\left(|\nabla w|^{2}+\left|w_{t}\right|^{2}\right) d x d y \leq C\left(\int_{0}^{T} \int_{0}^{1}\left|w_{x}(t, 1, y)\right|^{2} d y d t+\int_{0}^{T} \int_{0}^{1}\left|u_{y}(t, x, 1)\right|^{2} d x d t\right) .
$$

Indeed, (2.4) implies that $\mathcal{J}$ is coercive and ensures the existence of a minimizer, as stated in Theorem 2.2 .

Remark 2.1 For the continuous wave equation (1.2), the velocity of propagation of all waves is one and the bound of the minimal controllability time, $T>2 \sqrt{2}$, is exactly the minimum time that requires a wave, starting at any $x \in \Omega$ in any direction, to arrive to the controllability zone.

Remark 2.2 The control $(v, z)$ from Theorem 2.2 is characterized by the following two properties:

1. $(v, z)$ is a control for (1.2), or equivalently,

$$
\begin{aligned}
\int_{0}^{T} \int_{0}^{1} v(t, y) w_{x}(t, 1, y) d y d t & +\int_{0}^{T} \int_{0}^{1} z(t, x) w_{y}(t, x, 1) d x d t \\
& =<u^{1}, u(0)>-1,1-\int_{\Omega} u^{0}(x, y) w^{\prime}(0, x, y) d x d y
\end{aligned}
$$

for any $\left(w^{0}, w^{1}\right) \in H_{0}^{1}(\Omega) \times L^{2}(\Omega)$, being w the solution of the adjoint equation (2.2). 
2. There exists $\left(\widehat{u}^{0}, \widehat{w}^{1}\right) \in H_{0}^{1}(\Omega) \times L^{2}(\Omega)$ such that $v(t, y)=\widehat{u}_{x}(t, 1, y)$ and $z(t, x)=$ $\widehat{w}_{y}(t, x, 1)$, where $\left(\widehat{u}, \widehat{u}^{\prime}\right)$ is the solution of the adjoint system (2.2) with initial data $\left(\widehat{w}^{0}, \widehat{w}^{1}\right)$.

Much of our analysis will be based on Fourier expansion of solutions. Therefore, let us now introduce the eigenvalues of the wave equation $(2.2)$

$$
\lambda^{n m}=\operatorname{sgn}(n) \sqrt{n^{2}+m^{2}} \pi
$$

and the corresponding eigenfunctions

$$
\Psi^{n m}(x, y)=\sqrt{2}\left(\begin{array}{c}
\left(\mathbf{i} \lambda^{n m}\right)^{-1} \\
-1
\end{array}\right) \sin (n \pi x) \sin (m \pi y), \quad(n, m) \in \mathbb{Z}^{*} \times \mathbb{N}^{*}, \quad \mathbf{i}=\sqrt{-1}
$$

The sequence $\left(\Psi^{n m}\right)_{(n, m) \in \mathbb{Z}^{*} \times \mathbb{N}^{*}}$ forms an orthonormal basis in $H_{0}^{1}(\Omega) \times L^{2}(\Omega)$. Moreover,

$$
\left\|\Psi^{n m}\right\|_{L^{2}(\Omega) \times H^{-1}(\Omega)}=\frac{1}{\lambda^{n m}}
$$

The following characterization of any control of (1.2) in terms of the Fourier coefficients of initial data is useful.

Proposition 2.1 Given any $T>2 \sqrt{2}$ and $\left(u^{0}, u^{1}\right)=\sum_{(n, m) \in \mathbb{Z}^{*} \times \mathbb{N}^{*}} \alpha_{n m}^{0} \Phi^{n m} \in L^{2}(\Omega) \times H^{-1}(\Omega)$, $(v, z) \in\left[L^{2}((0, T) \times(0,1))\right]^{2}$ is a control for (1.2) if and only if, for all $(n, m) \in \mathbb{Z}^{*} \times \mathbb{N}^{*}$,

$$
\int_{0}^{T} e^{i \lambda^{n m} t}\left((-1)^{n} n \int_{0}^{1} v(t, y) \sin (m \pi y) d y+(-1)^{m} m \int_{0}^{1} z(t, x) \sin (n \pi x) d x\right) d t=\frac{\alpha_{n m}^{0}}{\sqrt{2} \pi}
$$

Proof: From the continuity of the linear form $\Lambda: H_{0}^{1}(\Omega) \times L^{2}(\Omega) \rightarrow \mathbb{C}$, defined by

$$
\begin{array}{r}
\Lambda\left(w^{0}, w^{1}\right)=\int_{0}^{T} \int_{0}^{1} v(t, y) w_{x}(t, 1, y) d y d t+\int_{0}^{T} \int_{0}^{1} z(t, x) w_{y}(t, x, 1) d x d t \\
-<u^{1}, w(0)>_{H^{-1}, H_{0}^{1}}+\int_{\Omega} u^{0}(x, y) w^{\prime}(0, x, y) d x d y
\end{array}
$$

it follows that $(2.5)$ holds for any $\left(w^{0}, w^{1}\right) \in H_{0}^{1}(\Omega) \times L^{2}(\Omega)$ if and only if it is verified on a basis of the space $H_{0}^{1}(\Omega) \times L^{2}(\Omega)$. Thus, by considering $\left(w^{0}, w^{1}\right)=\Psi^{n m}$ in (2.5), we obtain that the control $(v, z)$ drives to zero the initial data of $(1.2)$ if and only if $(2.8)$ is verified. 


\section{The semi-discrete problem}

In this section we introduce a suitable semi-discretization of the homogeneous adjoint equation (2.2). By minimizing the HUM functional corresponding to this semi-discrete system, a convergent sequence of discrete approximations $\left(v_{h}, z_{h}\right)_{h>0}$ of the HUM control $(v, z)$ of $(1.2)$ is obtained.

We introduce $N \in \mathbb{N}^{*}, h=1 /(N+1)$, we consider the points $\left(x_{i}, y_{j}\right)=(i h, j h), 0 \leq i, j \leq$ $N+1$ and we denote $w_{i j}=w\left(x_{i}, y_{j}\right)$.

Let us also introduce the new variable $\zeta(t, x, y)=w^{\prime}(t, x, y)$. Equation $(2.2)$ may be written in the following variational form:

$$
\left\{\begin{array}{l}
\text { Find }(w, \zeta)=(w, \zeta)(t, x, y) \quad \text { with }(w(t), \zeta(t)) \in H_{0}^{1}(\Omega) \times L^{2}(\Omega), \forall t \in(0, T) \text { and } \\
\frac{d}{d t} \int_{0}^{1} \int_{0}^{1} w(t, x, y) \psi(x, y) d x d y=\int_{0}^{1} \int_{0}^{1} \zeta(t, x, y) \psi(x, y) d x d y, \quad \forall \psi \in L^{2}(\Omega), \\
\frac{d}{d t}<\zeta(t, \cdot), \varphi>-1,1=\int_{0}^{1} \int_{0}^{1} \nabla w(t, x, y) \nabla \varphi(x, y) d x d y, \quad \forall \varphi \in H_{0}^{1}(\Omega), \\
w(T, x, y)=w^{0}(x, y), \zeta(T, x, y)=w^{1}(x, y), \quad \forall(x, y) \in \Omega .
\end{array}\right.
$$

We now discretize (3.1) by using a mixed finite element method

Let

$Q_{1}$ be the space of all polynomials of degree less or equal to one with respect to each one of the variables $x, y$ and $\mathbb{Q}_{0}$ the space of constant functions. We introduce the basis functions in the following way. For each $1 \leq i, j \leq N$, let $Q_{i, j}^{h}=\left(x_{i}, x_{i+1}\right) \times\left(y_{j}, y_{j+1}\right)$ be such that $\cup_{0 \leq i, j \leq N} Q_{i, j}^{h}=\Omega=(0,1)^{2}$ and define the functions

$$
\left\{\begin{array}{l}
\psi_{\left.i j\right|_{Q_{k l}^{h}} \in \mathbb{Q}_{0},} \quad \psi_{i j}= \begin{cases}\frac{1}{2} & \text { if }(x, y) \in Q_{i j}^{h} \cup Q_{i-1 j}^{h} \cup Q_{i j-1}^{h} \cup Q_{i-1 j-1}^{h}, \\
0 & \text { otherwise, }\end{cases} \\
\left.\varphi_{i j}\right|_{Q_{k l}^{h}} \in \mathbb{Q}_{1}, \quad \varphi_{i j}\left(x_{k}, y_{l}\right)=\delta_{i j}^{k l} .
\end{array}\right.
$$

The variational formulation (3.1) is then reduced to find

$$
w_{h}(t, x, y)=\sum_{i, j=1}^{N} w_{i j}(t) \varphi_{i j}(x, y) \quad \text { and } \quad \zeta_{h}(t, x, y)=\sum_{i, j=1}^{N} \zeta_{i j}(t) \psi_{i j}(x, y)
$$

that satisfy

$$
\left\{\begin{array}{lr}
\frac{d}{d t} \int_{0}^{1} \int_{0}^{1} w_{h}(t, x, y) \psi_{i j}(x, y) d x d y=\int_{0}^{1} \int_{0}^{1} \zeta_{h}(t, x) \psi_{i j}(x, y) d x d y, & \forall 1 \leq i, j \leq N, \\
\frac{d}{d t}<\zeta_{h}(t, \cdot), \varphi_{i j}>_{-1,1}=\int_{0}^{1} \int_{0}^{1} \nabla w_{h}(t, x, y) \nabla \varphi_{i j}(x, y) d x d y, & \forall 1 \leq i, j \leq N, \\
w_{h}(T, x, y)=w_{h}^{0}(x, y), \zeta_{h}(T, x, y)=w_{h}^{1}(x, y), & \forall(x, y) \in \Omega .
\end{array}\right.
$$


The variables $\zeta_{i j}$ may be eliminated in (3.2)-(3.3) leading to the following semi-discrete system for $w_{i j}(t)$, in $t \in(0, T)$ :

$$
\left\{\begin{array}{l}
\frac{h^{2}}{16}\left(4 w_{i j}^{\prime \prime}+2 w_{i+1 j}^{\prime \prime}+2 w_{i-1 j}^{\prime \prime}+2 w_{i j+1}^{\prime \prime}+2 w_{i j-1}^{\prime \prime}+w_{i+1 j+1}^{\prime \prime}+w_{i+1 j-1}^{\prime \prime}+w_{i-1 j+1}^{\prime \prime}+w_{i-1 j-1}^{\prime \prime}\right) \\
+\frac{1}{3}\left(8 w_{i j}-w_{i+1 j}-w_{i-1 j}-w_{i j+1}-w_{i j-1}-w_{i+1 j+1}-w_{i+1 j-1}-w_{i-1 j+1}-w_{i-1 j-1}\right)=0 \\
\text { for } 1 \leq i, j \leq N, \\
w_{i 0}=w_{i N+1}=0, \quad w_{0 j}=w_{N+1 j}=0, \quad \text { for } 0 \leq i \leq N+1, \\
w_{i j}(T)=w_{i j}^{0}, \quad w_{i j}^{\prime}(T)=w_{i j}^{1}, \text { for } 0 \leq i, j \leq N+1 .
\end{array}\right.
$$

The convergence of scheme (3.4) . We shall consider that the initial data are zero on the boundary of $\Omega$, which in the discrete equation corresponds to

$$
\left\{\begin{array}{l}
w_{0, j}^{0}=w_{0, j}^{1}=0, \quad w_{N+1, j}^{0}=w_{N+1, j}^{1}=0, \text { for } 0 \leq j \leq N+1, \\
w_{i, 0}^{0}=w_{i, 0}^{1}=0, \quad w_{i, N+1}^{0}=w_{i, N+1}^{1}=0, \text { for } 0 \leq i \leq N+1 .
\end{array}\right.
$$

The same property will be also satisfied by the corresponding solutions of (3.4).

If we denote the unknown

$$
W_{h}(t)=\left(w_{11}(t), w_{21}(t), \ldots, w_{N 1}, \ldots, w_{1 N}(t), w_{2 N}(t), \ldots, w_{N N}(t)\right)^{T}
$$

then equation (3.4) may be written in vectorial form as follows

$$
\left\{\begin{array}{l}
M_{h} W_{h}^{\prime \prime}(t)+K_{h} W_{h}(t)=0, \quad \text { for } t>0 \\
W_{h}(T)=W_{h}^{0}, W_{h}^{\prime}(T)=W_{h}^{1}
\end{array}\right.
$$

where $\left(W_{h}^{0}, W_{h}^{1}\right)=\left(w_{i j}^{0}, w_{i j}^{1}\right)_{1 \leq i, j \leq N} \in \mathbb{R}^{2 N^{2}}$ are the initial data and the corresponding solution of (3.4) is given by $\left(W_{h}, W_{h}^{\prime}\right)=\left(w_{i j}, w_{i j}^{\prime}\right)_{1 \leq i, j \leq N}$.

The entries of the block-three-diagonal matrices $M_{h}$ and $K_{h}$ belonging to $\mathcal{M}_{N^{2}}(\mathbb{R})$ may be easily deduced from (3.4).

\section{Properties of the semi-discrete system}

In this section we study some of the properties of the semi-discrete adjoint system (3.4), related to the controllability problem. More precisely, the aim of this section consists in giving a uniform (in h) observability inequality for (3.4). But before that, let us briefly explain why the semidiscretization introduced in this paper is likely to provide a uniform observability property rather than others, like the usual finite difference semi-discretization implemented 
As we have mentioned in Remark 2.1, in order to have an observability inequality for the continuous wave equation (2.2) of the type (2.4) it is necessary to consider $T>2 \sqrt{2}$. This is due to the finite velocity of propagation of waves. More precisely, a planar wave of the form $e^{\mathbf{i}(\xi \cdot(x, y)-\omega t)}$ propagates in any spatial direction $v=\left(v_{1}, v_{2}\right) \in \mathbb{R}^{2}$ with group velocity $\nabla_{\xi} \omega \cdot v$ where $\omega=|\xi|$. Let us denote

$$
\zeta=\min _{\xi \in \mathbb{R}^{2}} \max _{v \in \mathbb{R}^{2},|v|=1} \nabla_{\xi} \omega \cdot v=\min _{\xi \in \mathbb{R}^{2}}\left|\nabla_{\xi} \omega\right| .
$$

The observability time $T$ and $\zeta$ are inversely proportional. In our particular case $T>2$ diam $(\Omega) / \zeta$ (see $[10]$ ) and $\zeta=1$. Thus $T>2 \sqrt{2}$.

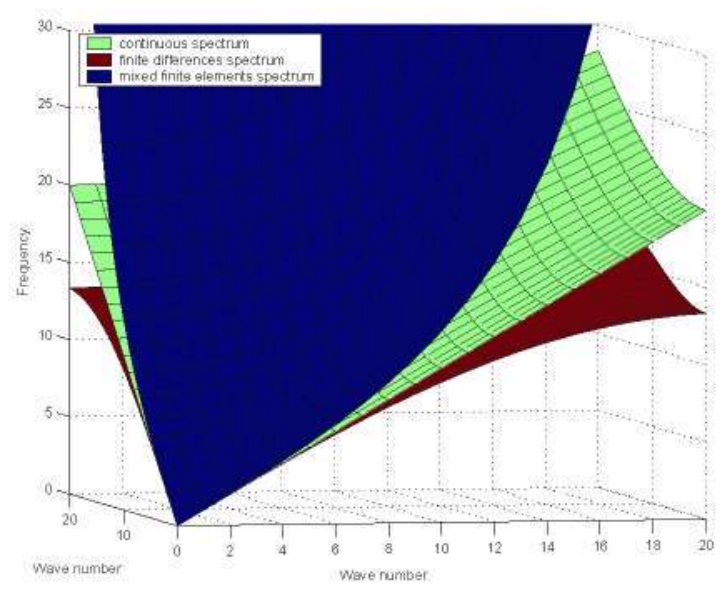

Figure 1: $\omega(\xi)$ with $\xi \in[0, \pi / h)^{2}$ and $h=1 / 21$ for the mixed finite element semi-discretization (upper surface), continuous wave equation (medium surface) and the usual finite differences semi-discretization (lower surface). We observe that the norm of the gradient $\left|\nabla_{\xi} \omega(\xi)\right|$ is always one in the continuous case, it is greater than one for the mixed finite element scheme and it becomes zero for the usual finite-difference scheme as $\xi$ approaches $(\pi / h, 0)$.

In a similar way, we may introduce the velocity of waves for the semi-discrete problem

. Let $w_{i j}=e^{\mathrm{i}\left(\xi \cdot\left(x_{i}, y_{j}\right)-\omega t\right)}, \quad \xi=\left(\xi_{1}, \xi_{2}\right) \in(-\pi / h, \pi / h)^{2}$ a discrete plane wave which propagates in any spatial direction $v=\left(v_{1}, v_{2}\right) \in \mathbb{R}^{2}$ with group velocity $\nabla_{\xi} \omega \cdot v$. In the mixed finite element method

$$
\omega=\omega_{m f e}(\xi)=\frac{2}{h} \sqrt{\tan ^{2}\left(\frac{\xi_{1} h}{2}\right)+\tan ^{2}\left(\frac{\xi_{2} h}{2}\right)+\frac{2}{3} \tan ^{2}\left(\frac{\xi_{1} h}{2}\right) \tan ^{2}\left(\frac{\xi_{2} h}{2}\right)},
$$


while for the finite difference method

$$
\omega=\omega_{f d}(\xi)=\frac{2}{h} \sqrt{\sin ^{2}\left(\frac{\xi_{1} h}{2}\right)+\sin ^{2}\left(\frac{\xi_{2} h}{2}\right)} .
$$

Note that $\zeta_{m f e}=\min _{\xi \in(-\pi / h, \pi / h)^{2}}\left|\nabla_{\xi} \omega_{m f e}\right|=1$ and $\zeta_{f d}=\min _{\xi \in(-\pi / h, \pi / h)^{2}}\left|\nabla_{\xi} \omega_{f d}\right|=\mathcal{O}(h)$. This is illustrated in Figure 1. Thus, the observability time $T$ can be uniformly bounded, in $h$, only for the mixed finite element method.

In the rest of this section we prove that indeed this property holds for system (3.4), using a different approach.

Since the matrices $M_{h}$ and $K_{h}$ are positive definite we may define the inner product

$$
<\left(f_{1}, f_{2}\right),\left(g_{1}, g_{2}\right)>_{0}=<K_{h} f_{1}, g_{1}>+<M_{h} f_{2}, g_{2}>
$$

for any $\left(f_{1}, f_{2}\right),\left(g_{1}, g_{2}\right) \in \mathbb{R}^{2 N^{2}}$, where $<,, \cdot>$ denotes the canonical inner product in $\mathbb{R}^{N^{2}}$. The corresponding norm will be denoted $\|\cdot\|_{0}$.

We introduce the following discrete version of the continuous energy of (2.2)

$$
E_{h}(t)=\frac{1}{2}\left\|\left(W_{h}, W_{h}^{\prime}\right)(t)\right\|_{0}^{2}
$$

The following proposition shows that, as in the corresponding continuous case, the energy $E_{h}$ defined by (4.3) is conserved along trajectories.

Proposition 4.1 For any $h>0$ and any solution of (3.4) the following holds

$$
E_{h}(t)=E_{h}(0), \quad \forall t>0 .
$$

Proof: Multiplying (3.6) by $W_{h}^{\prime}$, we obtain that

$$
0=<M_{h} W_{h}^{\prime \prime}, W_{h}^{\prime}>+<K_{h} W_{h}, W_{h}^{\prime}>=\frac{1}{2}\left[<M_{h} W_{h}^{\prime}, W_{h}^{\prime}>+<K_{h} W_{h}, W_{h}>\right]^{\prime}=\frac{d}{d t} E_{h}(t),
$$

and the proof finishes.

The following result shows that a discrete version of the observability inequality (2.4) is valid for the solutions of system (3.4).

Theorem 4.1 Given $T>2 \sqrt{3}$, there exists a constant $C(T)>0$ independent of the discretization step $h$, such that the following inequality holds

$$
\begin{aligned}
E_{h}(0) \leq & C(T) \frac{h}{2}\left\{\int _ { 0 } ^ { T } \left[\frac{1}{h^{2}}<C_{h} W_{N,}^{\prime}, W_{N .}^{\prime}>+\frac{1}{h^{2}}\left\langle C_{h} W_{. N}^{\prime}, W_{. N}^{\prime}>\right] d t-\right.\right. \\
& \left.-\int_{0}^{T}\left[\frac{1}{h^{2}}<B_{h} W_{N .}, W_{N}>+\frac{1}{h^{2}}<B_{h} W_{. N}, W_{. N}>\right] d t\right\},
\end{aligned}
$$

where $W_{N}=\left(w_{N j}\right)_{1 \leq j \leq N} \in \mathbb{R}^{N}$ and $W_{. N}=\left(w_{i N}\right)_{1 \leq i \leq N} \in \mathbb{R}^{N}$. 
Remark 4.1 The method used in the proof of the observability inequality (4.4) works only if $T>2 \sqrt{3}$. Probably this time is not sharp and the same is true for $T>2 \sqrt{2}$ which is the necessary and sufficient time condition for controllability in the continuous case (see Remark 2.1).

The proof of Theorem 4.1 is technical and it is given in the Appendix.

\section{Construction of the discrete approximations}

In this section we explicitly construct a sequence of approximations $\left(v_{h}, z_{h}\right)_{h>0}$ of the HUM control $(v, z)$ of $(1.2)$. This will be done by minimizing the HUM functional of the semi-discrete adjoint system (3.4).

Suppose that $\left(U_{h}^{0}, U_{h}^{1}\right)=\left(u_{j}^{0}, u_{j}^{1}\right)_{1 \leq j \leq N} \in \mathbb{R}^{2 N^{2}}$ is a discretization of the continuous initial data of (1.2) to be controlled. We define the functional $\mathcal{J}_{h}: \mathbb{R}^{2 N^{2}} \rightarrow \mathbb{R}$,

$$
\begin{aligned}
\mathcal{J}_{h}\left(\left(W_{h}^{0}, W_{h}^{1}\right)\right)= & -<\left(-K_{h}^{-1} M_{h} U_{h}^{1}, U_{h}^{0}\right),\left(W_{h}(0), W_{h}^{\prime}(0)\right)>_{0} \\
& +\frac{1}{2 h} \int_{0}^{T}\left[<C_{h} W_{N}^{\prime}, W_{N}^{\prime}>+<C_{h} W_{. N}^{\prime}, W_{. N}^{\prime}>\right] d t \\
& +\frac{1}{2 h} \int_{0}^{T}\left[<B_{h} W_{N}, W_{N}>+<B_{h} W_{. N}, W_{. N}>\right] d t
\end{aligned}
$$

where $\left(W_{h}, W_{h}^{\prime}\right)$ is the solution of $(3.6)$ with initial data $\left(W_{h}^{0}, W_{h}^{1}\right) \in \mathbb{R}^{2 N^{2}}$, and we have noted $W_{N,}=\left(w_{N j}\right)_{1 \leq j \leq N} \in \mathbb{R}^{N}$ and $W_{. N}=\left(w_{i N}\right)_{1 \leq i \leq N} \in \mathbb{R}^{N}$.

We show now that $\mathcal{J}_{h}$ has a minimizer $\left(\widehat{W}_{h}^{0}, \widehat{W}_{h}^{1}\right)$.

Theorem 5.1 For any $T>2 \sqrt{3}$ the functional $\mathcal{J}_{h}$ (5.1) has an unique minimizer $\left(\widehat{W}_{h}^{0}, \widehat{W}_{h}^{1}\right)$.

Proof: Since $\mathcal{J}_{h}$ is continuous, convex and defined in a finite dimensional space, the theorem is proved if we show that $\mathcal{J}_{h}$ is coercive. This is a consequence of (4.4). More precisely,

$$
\begin{aligned}
& \mathcal{J}_{h}\left(W_{h}^{0}, W_{h}^{1}\right) \geq \frac{h}{32} \int_{0}^{T}\left(\sum_{j=0}^{N}\left|w_{N j+1}^{\prime}(t)+w_{N j}^{\prime}(t)\right|^{2}+\sum_{i=0}^{N}\left|w_{i+1 N}^{\prime}(t)+w_{i N}^{\prime}(t)\right|^{2}\right) d t \\
& +\frac{1}{6 h} \int_{0}^{T}\left(\sum_{j=0}^{N}\left|w_{N j+1}(t)+w_{N j}(t)\right|^{2}+\sum_{i=0}^{N}\left|w_{i+1 N}(t)+w_{i N}(t)\right|^{2}\right) d t \\
& \quad-\frac{1}{6 h} \int_{0}^{T}\left(\sum_{j=0}^{N}\left|w_{N j}(t)\right|^{2}+\sum_{i=0}^{N}\left|w_{i N}(t)\right|^{2}\right) d t-\left\|\left(-K_{h}^{-1} M_{h} U_{h}^{1}, U_{h}^{0}\right)\right\|_{0}\left\|\left(W_{h}(0), W_{h}^{\prime}(0)\right)\right\|_{0} \\
& \geq C(T)\left\|\left(W_{h}^{0}, W_{h}^{1}\right)\right\|_{0}^{2}-\left\|\left(-K_{h}^{-1} M_{h} U_{h}^{1}, U_{h}^{0}\right)\right\|_{0}\left\|\left(W_{h}^{0}, W_{h}^{1}\right)\right\|_{0},
\end{aligned}
$$


and therefore

$$
\lim _{\left\|\left(W_{h}^{0}, W_{h}^{1}\right)\right\|_{0} \rightarrow \infty} \mathcal{J}\left(W_{h}^{0}, W_{h}^{1}\right)=\infty
$$

Remark 5.1 The main tool in the proof of the previous result is the observability inequality (4.4) stated in Theorem 4.1. It ensures the coercivity of $\mathcal{J}$ and consequently the existence of a minimizer. Moreover, as we shall see in Theorem 6.1, the constant $C(T)$ appearing in (4.4) is an upper bound for the sequences of minimizers and of controls.

Let $\left(\widehat{W}_{h}^{0}, \widehat{W}_{h}^{1}\right)$ be the minimizer of the functional $\mathcal{J}_{h}$ given by Theorem 5.1. We define $v_{h}=$ $\left(v_{h, j}\right)_{1 \leq j \leq N} \in L^{2}\left(0, T ; \mathbb{R}^{N}\right)$ and $z_{h}=\left(z_{h, i}\right)_{1 \leq i \leq N} \in L^{2}\left(0, T ; \mathbb{R}^{N}\right)$ by

$$
v_{h, j}(t)=-\frac{\widehat{w}_{N j}}{h}, \quad z_{h, i}(t)=-\frac{\widehat{w}_{i N}}{h}, \quad \forall 1 \leq i, j \leq N,
$$

where $\left(\widehat{W}_{h}, \widehat{W}_{h}^{\prime}\right)$ is the solution of (3.6) with initial data $\left(\widehat{W}_{h}^{0}, \widehat{W}_{h}^{1}\right)$.

Our aim is to show that the sequence $\left(v_{h}, z_{h}\right)_{h>0}$ converges to a control $(v, z)$ of the continuous equation $(1.2)$. Since $v_{h}$ and $z_{h}$ belong to $L^{2}\left(0, T ; \mathbb{R}^{N}\right)$ whereas $v$ and $z$ are in $L^{2}\left(0, T ; L^{2}(0,1)\right)$ the convergence is stated in terms of the Fourier coefficients. This is done in the next section.

In the rest of this section we introduce the eigenfunctions and the eigenvalues of the semidiscrete problem (3.6). Let $\mathcal{I}_{N}=\left\{(n, m) \in \mathbb{Z}^{*} \times \mathbb{N}^{*}: 1 \leq|n| \leq N, 1 \leq m \leq N\right\}$.

Lemma 5.1 The eigenvalues $\lambda_{h}^{n m},(n, m) \in \mathcal{I}_{N}$, of the semi-discrete problem (3.6) are given by

$$
\lambda_{h}^{n m}=\operatorname{sgn}(n) \frac{2}{h} \sqrt{\tan ^{2}\left(\frac{m \pi h}{2}\right)+\tan ^{2}\left(\frac{n \pi h}{2}\right)+\frac{2}{3} \tan ^{2}\left(\frac{m \pi h}{2}\right) \tan ^{2}\left(\frac{n \pi h}{2}\right)} .
$$

The corresponding eigenfunctions are

$$
\Psi_{h}^{n m}=\frac{\sqrt{2}}{\cos \left(\frac{n \pi h}{2}\right) \cos \left(\frac{m \pi h}{2}\right)}\left(\begin{array}{c}
\left(i \lambda_{h}^{n m}\right)^{-1} \Phi_{h}^{n m} \\
-\Phi_{h}^{n m}
\end{array}\right), \quad \forall(n, m) \in \mathcal{I}_{N},
$$

where $\Phi_{h}^{n m}=\left(\phi_{h}^{n} \sin (p m \pi h)\right)_{1 \leq p \leq N} \in \mathbb{R}^{N^{2}}$ and $\phi_{h}^{n}=(\sin (j n \pi h))_{1 \leq j \leq N} \in \mathbb{R}^{N}$.

A straightforward computation shows that $\left(\Psi_{h}^{n m}\right)_{(n, m) \in \mathcal{I}_{N}}$ constitutes an orthonormal basis in $R^{2 N^{2}}$ with respect to the inner product $<\cdot, \cdot>_{0}$.

For any $\left(f^{1}, f^{2}\right),\left(g^{1}, g^{2}\right) \in \mathbb{R}^{2 N^{2}}$ we introduce the notations

$$
\begin{gathered}
<\left(f^{1}, f^{2}\right),\left(g^{1}, g^{2}\right)>_{-1}=<\left(-K_{h}^{-1} M_{h} f^{2}, f^{1}\right),\left(-K_{h}^{-1} M_{h} g^{2}, g^{1}\right)>_{0}, \\
\left\|\left(f^{1}, f^{2}\right)\right\|_{-1}=\left\|\left(-K_{h}^{-1} M_{h} f^{2}, f^{1}\right)\right\|_{0} .
\end{gathered}
$$

Remark that $<\cdot, \cdot>_{-1}$ is an inner product and $\|\cdot\|_{-1}$ is a norm on $\mathbb{R}^{2 N^{2}}$. 


\section{Convergence of the discrete approximations}

In this section we prove the weak convergence of the sequence $\left(v_{h}, z_{h}\right)_{h>0}$ to the HUM control of the continuous equation (1.2). Let us first show the following boundedness property of the initial data from which $\left(v_{h}, z_{h}\right)$ were constructed.

Theorem 6.1 Assume that $T>2 \sqrt{3}$. The sequence of minimizers of $\mathcal{J}_{h}$ given by Theorem 5.1 . $\left(\widehat{W}_{h}^{0}, \widehat{W}_{h}^{1}\right)_{h>0}$, verify

$$
\left\|\left(\widehat{W}_{h}^{0}, \widehat{W}_{h}^{1}\right)\right\|_{0} \leq \frac{1}{C}\left\|\left(-K_{h}^{-1} M_{h} U_{h}^{1}, U_{h}^{0}\right)\right\|_{0}
$$

where $C=C(T)$ is the observability constant of (4.4) which is independent of $h$.

If the sequence of discretizations $\left(U_{h}^{0}, U_{h}^{1}\right)_{h>0}$ is uniformly bounded in the $\|\cdot\|_{-1}-$ norm then the sequence $\left(\widehat{W}_{h}^{0}, \widehat{W}_{h}^{1}\right)_{h>0}$ is bounded in the $\|\cdot\|_{0}-$ norm.

Proof: From the observability inequality (4.4) we have that

$$
\begin{aligned}
& \left.\left.C\left\|\left(\widehat{W}_{h}^{0}, \widehat{W}_{h}^{1}\right)\right\|_{0}^{2} \leq \frac{h}{2} \int_{0}^{T}\left[<C_{h} v_{h}^{\prime}, v_{h}^{\prime}\right\rangle+<C_{h} z_{h}^{\prime}, z_{h}^{\prime}\right\rangle\right] d t \\
& -\frac{h}{2} \int_{0}^{T}\left[<B_{h} v_{h}, v_{h}>+<B_{h} z_{h}, z_{h}>\right] d t \\
& \left.=\mathcal{J}_{h}\left(\widehat{W}_{h}^{0}, \widehat{W}_{h}^{1}\right)+<\left(-K_{h}^{-1} M_{h} U_{h}^{1}, U_{h}^{0}\right), \widehat{W}_{h}(0), \widehat{W}_{h}^{\prime}(0)\right)>_{0} \text {. }
\end{aligned}
$$

Now, since $\mathcal{J}_{h}\left(\widehat{W}_{h}^{0}, \widehat{W}_{h}^{1}\right) \leq \mathcal{J}_{h}(0,0)=0$, it follows that

$$
\begin{array}{r}
C\left\|\left(\widehat{W}_{h}^{0}, \widehat{W}_{h}^{1}\right)\right\|_{0}^{2} \leq<\left(-K_{h}^{-1} M_{h} U_{h}^{1}, U_{h}^{0}\right),\left(\widehat{W}_{h}(0), \widehat{W_{h}^{\prime}}(0)\right)>_{0} \\
\leq\left\|\left(-K_{h}^{-1} M_{h} U_{h}^{1}, U_{h}^{0}\right)\right\|_{0}\left\|\left(W_{h}(0), W_{h}^{\prime}(0)\right)\right\|_{0} \\
\quad=\left\|\left(-K_{h}^{-1} M_{h} U_{h}^{1}, U_{h}^{0}\right)\right\|_{0}\left\|\left(W_{h}^{0}, W_{h}^{1}\right)\right\|_{0},
\end{array}
$$

which is equivalent to (6.1).

Remark 6.1 Theorem 6.1 shows that the sequence of initial data $\left(\widehat{W}_{h}^{0}, \widehat{W}_{h}^{1}\right)_{h>0}$ which give $\left(v_{h}, z_{h}\right)$ is uniformly bounded in $h$ for the $\|\cdot\|_{0}-$ norm if the sequence of discretizations $\left(U_{h}^{0}, U_{h}^{1}\right)_{h>0}$ is bounded in the $\|\cdot\|_{-1}-$ norm. The sequences $\left(v_{h}, z_{h}\right)_{h>0}$ verifies the following inequality

$$
\begin{gathered}
\frac{h}{2} \int_{0}^{T}\left[<C_{h} v_{h}^{\prime}, v_{h}^{\prime}>+<C_{h} \tilde{z}_{h}^{\prime}, z_{h}^{\prime}>-<B_{h} v_{h}, v_{h}>-<B_{h} z_{h}, z_{h}>\right] d t \\
\leq \frac{1}{C}\left\|\left(-K_{h}^{-1} M_{h} U_{h}^{1}, U_{h}^{0}\right)\right\|_{0}^{2}=\frac{1}{C}\left\|\left(U_{h}^{0}, U_{h}^{1}\right)\right\|_{-1}^{2} .
\end{gathered}
$$




\subsection{Weak convergence of the approximations}

Assume that the sequence of discretizations of the continuous initial data on $(1.2),\left(U_{h}^{0}, U_{h}^{1}\right)_{h>0}$, converges weakly to $\left(u^{0}, u^{1}\right)$ in $L^{2}(\Omega) \times H^{-1}(\Omega)$. This should be understood in the sense of the convergence of the Fourier coefficients. More precisely, if

$$
\left(U_{h}^{0}, U_{h}^{1}\right)=\sum_{(n, m) \in \mathcal{I}_{N}} \alpha_{n m}^{h} \Phi_{h}^{n m}, \quad\left(u^{0}, u^{1}\right)=\sum_{(n, m) \in \mathbb{Z}^{*} \times \mathbb{N}^{*}} \alpha_{n m} \Phi^{n m}
$$

then the following weak convergence holds in $\ell^{2}$

$$
\left(\frac{\alpha_{n m}^{h}}{\lambda_{h}^{n m}}\right)_{(n, m) \in \mathcal{I}_{N}} \rightarrow\left(\frac{\alpha_{n m}}{\lambda^{n m}}\right)_{(n, m) \in \mathbb{Z}^{*} \times \mathbb{N}^{*}}, \text { when } h \rightarrow 0 .
$$

Now, assume that the minimizer $\left(\widehat{W}_{h}^{0}, \widehat{W}_{h}^{1}\right)$ has the following expansion

$$
\left(\widehat{W}_{h}^{0}, \widehat{W}_{h}^{1}\right)=\sum_{(n, m) \in \mathcal{I}_{N}} a_{n m}^{h} \Psi_{h}^{n m}
$$

Inequality (6.1) is equivalent to

$$
\sum_{(n, m) \in \mathcal{I}_{N}}\left|a_{n m}^{h}\right|^{2}=\left\|\left(\widehat{W}_{h}^{0}, \widehat{W}_{h}^{1}\right)\right\|_{0}^{2} \leq \frac{1}{C^{2}}\left\|\left(-K_{h}^{-1} M_{h} U_{h}^{1}, U_{h}^{0}\right)\right\|_{0}^{2}=\frac{1}{C^{2}} \sum_{(n, m) \in I_{N}}\left|\frac{\alpha_{n m}^{h}}{\lambda_{h}^{n m}}\right|^{2} .
$$

Here, the right hand side is bounded due to the weak convergence stated in (6.3). Hence, the sequence of Fourier coefficients $\left(a_{n m}^{h}\right)_{(n, m) \in \mathcal{I}_{N}}$ is bounded in $\ell^{2}$ and there exists a subsequence, denoted in the same way, and $\left(a_{n m}\right)_{(n, m) \in \mathbb{Z}^{*} \times \mathbb{N}^{*}} \in \ell^{2}$ such that

$$
\left(a_{n m}^{h}\right)_{(n, m) \in \mathcal{I}_{N}} \rightarrow\left(a_{n m}\right)_{(n, m) \in \mathbb{Z}^{*} \times \mathbb{N}^{*}} \text { in } \ell^{2} \text { when } h \rightarrow 0 .
$$

Let us now introduce the continuous initial data.

$$
\left(\widehat{w}^{0}, \widehat{w}^{1}\right)=\sum_{(n, m) \in \mathbb{Z}^{*} \times \mathbb{N}^{*}} a_{n m} \Psi^{n m} \in H_{0}^{1}(\Omega) \times L^{2}(\Omega),
$$

and the corresponding solution $\left(\widehat{u}, \widehat{u}^{\prime}\right) \in C\left([0, T] ; H_{0}^{1}(\Omega) \times L^{2}(\Omega)\right)$. We have that

$$
\begin{aligned}
& \widehat{w}_{x}(t, 1, y)=\sum_{m \in \mathbb{N}^{*}}\left(\sum_{n \in \mathbb{Z}^{*}} i a_{n m}(-1)^{n+1} \frac{\sqrt{2} n \pi}{\lambda^{n m}} e^{i \lambda^{n m} t}\right) \sin (m \pi y):=v, \\
& \widehat{w}_{y}(t, x, 1)=\sum_{n \in \mathbb{Z}^{*}}\left(\sum_{m \in \mathbb{N}^{*}} i a_{n m}(-1)^{m+1} \frac{\sqrt{2} m \pi}{\lambda^{n m}} e^{i \lambda^{n m} t}\right) \sin (n \pi x):=w
\end{aligned}
$$

If $\left(\widehat{W}_{h}, \widehat{W}_{h}^{\prime}\right)$ is the corresponding solution of (3.6) with initial data $\left(\widehat{W}_{h}^{0}, \widehat{W}_{h}^{1}\right)$, it follows that

$$
\begin{aligned}
& v_{h}=\sum_{1 \leq m \leq N}\left(\sum_{1 \leq|n| \leq N} i a_{n m}^{h}(-1)^{n+1} \frac{\sqrt{2}}{\lambda_{h}^{n m} \cos \left(\frac{n \pi h}{2}\right) \cos \left(\frac{m \pi n h}{2}\right)} \sin (n \pi h) e^{i \lambda_{h}^{n m} t}\right) \phi_{h}^{m}, \\
& \tilde{z} h_{h}=\sum_{1 \leq|n| \leq N}\left(\sum_{1 \leq m \leq N} i a_{n m}^{h}(-1)^{m+1} \frac{\sqrt{2}}{\lambda_{h}^{m m} \cos \left(\frac{n \pi h}{2}\right) \cos \left(\frac{m \pi h}{2}\right)} \sin (m \pi h) e^{i \lambda_{h}^{n m} t}\right) \phi_{h}^{n} .
\end{aligned}
$$


We denote

$$
\begin{aligned}
& b_{m}^{h}= \begin{cases}\sum_{1 \leq|n| \leq N} i a_{n m}^{h}(-1)^{n+1} \frac{\sqrt{2}}{\lambda_{h}^{n m} \cos \left(\frac{n \pi h}{2}\right) \cos \left(\frac{m \pi h}{2}\right)} \sin (n \pi h) e^{i \lambda_{h}^{n m} t}, & \text { if } 1 \leq m \leq N, \\
0, & \text { if } m>N,\end{cases} \\
& b_{m}=\sum_{n \in \mathbb{Z}^{*}} i a_{n m}(-1)^{n+1} \frac{\sqrt{2} n \pi}{\lambda^{n m}} e^{i \lambda^{n m} t} \\
& d_{n}^{h}=\left\{\begin{array}{ll}
\sum_{1 \leq m \leq N} i a_{n m}^{h}(-1)^{m+1} \frac{\sqrt{2}}{\lambda_{h}^{n m} \cos \left(\frac{n \pi h}{2}\right) \cos \left(\frac{m \pi h}{2}\right)} \sin (m \pi h) e^{i \lambda_{h}^{n m} t}, & \text { if } 1 \leq|n| \leq N, \\
0, & \text { if }|n|>N,
\end{array},\right. \\
& d_{n}=\sum_{m \in \mathbb{N}^{*}} i a_{n m}(-1)^{m+1} \frac{\sqrt{2} m \pi}{\lambda^{n m}} e^{i \lambda^{m m} t}
\end{aligned}
$$

Theorem 6.2 Assume that the sequence of discretizations $\left(U_{h}^{0}, U_{h}^{1}\right)_{h>0}$ converges weakly to $\left(u^{0}, u^{1}\right)$ in the sense of (6.3). The following convergencies hold weakly in $L^{2}\left(0, T ; \ell^{2}\right)$ when $h$ tends to zero

$$
\begin{gathered}
\left(b_{m}^{h}\right)_{m \in \mathbb{N}^{*}} \rightarrow\left(b_{m}\right)_{m \in \mathbb{N}^{*}}, \quad\left(d_{n}^{h}\right)_{n \in \mathbb{Z}^{*}} \rightarrow\left(d_{n}\right)_{n \in \mathbb{Z}^{*}}, \\
\left(h\left(b_{m}^{h}\right)^{\prime}\right)_{m \in \mathbb{N}^{*}} \rightarrow 0, \quad\left(h\left(d_{n}^{h}\right)^{\prime}\right)_{n \in \mathbb{Z}^{*}} \rightarrow 0 .
\end{gathered}
$$

In particular $\left(v_{h}, z_{h}\right)_{h>0}$ converges weakly to $(v, z)$ in $\left[L^{2}((0, T) \times(0,1))\right]^{2}$.

Proof: We show the first convergence, the other ones being similar. Let us introduce

$$
\begin{aligned}
\tilde{b}_{m}^{h}(t) & =\sum_{1 \leq|n| \leq N} i a_{n m}^{h}(-1)^{n+1} \frac{\sqrt{2} \sin (n \pi h)}{\lambda_{h}^{n m} \cos \left(\frac{n \pi h}{2}\right) \cos \left(\frac{m \pi h}{2}\right)} \frac{1}{\left(\lambda_{h}^{n m}\right)^{2}} e^{i \lambda_{h}^{n m} t}, \\
\tilde{b}_{m}(t) & =\sum_{n \in \mathbb{Z}^{*}} i a_{n m}(-1)^{n+1} \frac{\sqrt{2 n \pi}}{\lambda^{n m}} \frac{1}{\left(\lambda^{n m}\right)^{2}} e^{i \lambda^{n m} t} .
\end{aligned}
$$

The convergence $\left(b_{m}^{h}\right)_{m \in \mathbb{N}^{*}} \rightarrow\left(b_{m}\right)_{m \in \mathbb{N}^{*}}$ is proved if we show that

$$
\int_{0}^{T} \sum_{m \geq 1}\left|\tilde{b}_{m}^{h}(t)-\tilde{b}_{m}(t)\right|^{2} d t \longrightarrow 0 \quad \text { when } \quad h \rightarrow 0 .
$$

In order to prove (6.7) we consider an arbitrary $\varepsilon>0$ and show that there exists $N$ sufficiently large such that

$$
\int_{0}^{T} \sum_{m>N}\left|\tilde{b}_{m}(t)\right|^{2} d t \leq \frac{\varepsilon}{2}
$$

and

$$
\int_{0}^{T} \sum_{1 \leq m \leq N}\left|\tilde{b}_{m}^{h}(t)-\tilde{b}_{m}(t)\right|^{2} d t \leq \frac{\varepsilon}{2}
$$


Remark that (6.8) and (6.9) imply (6.7) immediately.

To prove (6.8) note that, since $\left(a_{n m}\right) \in \ell^{2}$, there exists $N_{1}>0$ independent of $h$ such that, for any $N>N_{1}$, we have

$$
\begin{gathered}
\int_{0}^{T} \sum_{m>N}\left|\tilde{b}_{m}(t)\right|^{2} d t \leq \int_{0}^{T} \sum_{m>N}\left(\sum_{n \in \mathbb{Z}^{*}} \frac{1}{\left|\lambda^{n m}\right|^{4}}\right)\left(\sum_{n \in \mathbb{Z}^{*}}\left|i a_{n m}(-1)^{n+1} \frac{\sqrt{2} n \pi}{\lambda^{n m}} e^{i \lambda^{n m} t}\right|^{2}\right) d t \\
\quad \leq 2\left(\sum_{m>N} \sum_{n \in \mathbb{Z}^{*}} \frac{1}{\left|\lambda^{n m}\right|^{4}}\right) \int_{0}^{T}\left(\sum_{m>N} \sum_{n \in \mathbb{Z}^{*}}\left|a_{n m}\right|^{2} d t\right) \leq c T \sum_{m>N} \sum_{n \in \mathbb{Z}^{*}}\left|a_{n m}\right|^{2} \leq \frac{\xi}{2}
\end{gathered}
$$

Let us now show that, for $h$ sufficiently small (or, equivalently, for $N$ sufficiently large), (6.9) also holds. We have that

$$
\begin{gathered}
\frac{1}{2} \sum_{1 \leq m \leq N}\left|\tilde{b}_{m}^{h}-\tilde{b}_{m}\right|^{2} \leq \\
\sum_{1 \leq m \leq N}\left|\sum_{1 \leq|n| \leq N}(-1)^{n+1} i a_{n m}^{h}\left(\frac{\sqrt{2} \sin (n \pi h)}{\lambda_{h}^{n m} \cos \left(\frac{n \pi h}{2}\right) \cos \left(\frac{m \pi h}{2}\right)} \frac{1}{\left(\lambda_{h}^{n m}\right)^{2}} e^{i \lambda_{h}^{m m} t}-\frac{\sqrt{2} n \pi}{\lambda^{n m}} \frac{1}{\left(\lambda^{n m}\right)^{2}} e^{i \lambda^{n m} t}\right)\right|^{2} \\
+\sum_{1 \leq m \leq N}\left|\sum_{1 \leq|n| \leq N} i(-1)^{n+1}\left(a_{n m}^{h}-a_{n m}\right) \frac{\sqrt{2} n \pi}{\lambda^{n m}} \frac{1}{\left(\lambda^{n m}\right)^{2}} e^{i \lambda^{n m m} t}\right|^{2}
\end{gathered}
$$

According to the weak convergence of the sequence $\left(a_{n m}^{h}\right)_{n m}$ to $\left(a_{n m}\right)_{n m}$ and the presence of the weights $1 /\left(\lambda^{n m}\right)^{2}$, for $h$ sufficiently small,

$$
\sum_{1 \leq m \leq N}\left|\sum_{1 \leq|n| \leq N} i(-1)^{n+1}\left(a_{n m}^{h}-a_{n m}\right) \frac{\sqrt{2} n \pi}{\lambda^{n m}} \frac{1}{\left(\lambda^{n m}\right)^{2}} e^{i \lambda^{n m m} t}\right|^{2} \leq \frac{\varepsilon}{4} .
$$

On the other hand,

$$
\begin{aligned}
& \sum_{1 \leq m \leq N}\left|\sum_{1 \leq|n| \leq N}(-1)^{n+1} i a_{n m}^{h}\left(\frac{\sqrt{2} \sin (n \pi h)}{\lambda_{h}^{n m} \cos \left(\frac{n \pi h}{2}\right) \cos \left(\frac{m \pi h}{2}\right)} \frac{e^{i \lambda_{h}^{n m} t}}{\left(\lambda_{h}^{n m}\right)^{2}}-\frac{\sqrt{2} n \pi}{\lambda^{n m}} \frac{e^{i \lambda^{n m} t}}{\left(\lambda^{n m}\right)^{2}}\right)\right|^{2} \\
\leq & \sum_{1 \leq m \leq N}\left(\sum_{1 \leq|n| \leq N}\left|a_{n m}^{h}\right|^{2} \sum_{1 \leq|n| \leq N}\left|\frac{\sqrt{2} \sin (n \pi h)}{\lambda_{h}^{n m} \cos \left(\frac{n \pi h}{2}\right) \cos \left(\frac{m \pi h}{2}\right)} \frac{e^{i \lambda_{h}^{n m} t}}{\left(\lambda_{h}^{n m}\right)^{2}}-\frac{\sqrt{2} n \pi}{\lambda^{n m}} \frac{e^{i \lambda^{n m} t}}{\left(\lambda^{n m}\right)^{2}}\right|^{2}\right) .
\end{aligned}
$$

Since $\left(a_{n m}^{h}\right)_{n m}$ is bounded in $\ell^{2}$ there exists $c>0$ such that

$$
\sum_{1 \leq|n| \leq N}\left|a_{n m}^{h}\right|^{2} \leq \sum_{1 \leq m \leq N} \sum_{1 \leq|n| \leq N}\left|a_{n m}^{h}\right|^{2} \leq c,
$$

and (6.9) follows if we prove that

$$
\sum_{1 \leq m \leq N} \sum_{1 \leq|n| \leq N}\left|\frac{\sqrt{2} \sin (n \pi h)}{\lambda_{h}^{n m} \cos \left(\frac{n \pi h}{2}\right) \cos \left(\frac{m \pi h}{2}\right)} \frac{1}{\left(\lambda_{h}^{n m}\right)^{2}} e^{i \lambda_{h}^{n m} t}-\frac{\sqrt{2} n \pi}{\lambda^{n m}} \frac{1}{\left(\lambda^{n m}\right)^{2}} e^{i \lambda^{n m} t}\right|^{2} \leq \frac{\varepsilon}{4 c} .
$$


Note that

$$
\max \left\{\left|\frac{\sqrt{2} \sin (n \pi h)}{\lambda_{h}^{n m} \cos \left(\frac{n \pi h}{2}\right) \cos \left(\frac{m \pi h}{2}\right)}\right|, \frac{\sqrt{2} n \pi}{\lambda^{n m}}\right\} \leq \sqrt{3}
$$

and thus there exists $n_{\varepsilon}>0$ independent of $h$ such that

$$
\begin{gathered}
\sum_{1 \leq m \leq N} \sum_{n_{\varepsilon}+1 \leq|n| \leq N}\left|\frac{\sqrt{2} \sin (n \pi h)}{\lambda_{h}^{n m} \cos \left(\frac{n \pi h}{2}\right) \cos \left(\frac{m \pi h}{2}\right)} \frac{1}{\left(\lambda_{h}^{n m}\right)^{2}} e^{i \lambda_{h}^{n m} t}-\frac{\sqrt{2} n \pi}{\lambda^{n m}} \frac{1}{\left(\lambda^{n m}\right)^{2}} e^{i \lambda^{n m} t}\right|^{2} \\
+\sum_{n_{\varepsilon}+1 \leq m \leq N} \sum_{1 \leq|n| \leq n_{\varepsilon}}\left|\frac{\sqrt{2} \sin (n \pi h)}{\lambda_{h}^{n m} \cos \left(\frac{n \pi h}{2}\right) \cos \left(\frac{m \pi h}{2}\right)} \frac{1}{\left(\lambda_{h}^{n m}\right)^{2}} e^{i \lambda_{h}^{n m} t}-\frac{\sqrt{2} n \pi}{\lambda^{n m}} \frac{1}{\left(\lambda^{n m}\right)^{2}} e^{i \lambda^{n m} t}\right|^{2} \\
\leq 6 \sum_{1 \leq m \leq N} \sum_{n_{\varepsilon}+1 \leq|n| \leq N} \frac{1}{\left(\lambda^{n m}\right)^{2}}+6 \sum_{n_{\varepsilon}+1 \leq m \leq N} \sum_{1 \leq|n| \leq n_{\varepsilon}} \frac{1}{\left(\lambda^{n m}\right)^{2}} \leq \frac{\xi}{8 c} .
\end{gathered}
$$

Let us now analyze the case $1 \leq m,|n| \leq n_{\varepsilon}$. Since $\lambda_{h}^{n m} \rightarrow \lambda^{n m}$ when $h$ tends to zero, it follows that, for $h$ sufficiently small,

$$
\begin{aligned}
& \left|\frac{\sqrt{2} \sin (n \pi h)}{\lambda_{h}^{n m} \cos \left(\frac{n \pi h}{2}\right) \cos \left(\frac{m \pi h}{2}\right)} \frac{1}{\left(\lambda_{h}^{n m}\right)^{2}} e^{i \lambda_{h}^{n m} t}-\frac{\sqrt{2} n \pi}{\lambda^{n m}} \frac{1}{\left(\lambda^{n m}\right)^{2}} e^{i \lambda^{n m} t}\right|^{2} \\
& \leq \frac{\sqrt{2}}{\left(\lambda^{n m}\right)^{4}}\left|\frac{\frac{\sin (n \pi h)}{n \pi} \lambda^{n m}}{\lambda_{h}^{n m} \cos \left(\frac{n \pi h}{2}\right) \cos \left(\frac{m \pi h}{2}\right)} \frac{\left(\lambda^{n m}\right)^{2}}{\left(\lambda_{h}^{n m}\right)^{2}} e^{i\left(\lambda_{h}^{n m}-\lambda^{n m}\right) t}-1\right|^{2} \leq \frac{\varepsilon}{8 c n n_{\varepsilon}^{2}} .
\end{aligned}
$$

Consequently

$$
\sum_{1 \leq m \leq n_{\varepsilon}} \sum_{1 \leq|n| \leq n_{\varepsilon}}\left|\frac{\sqrt{2} \sin (n \pi h)}{\lambda_{h}^{n m} \cos \left(\frac{n \pi h}{2}\right) \cos \left(\frac{m \pi h}{2}\right)} \frac{1}{\left(\lambda_{h}^{n m}\right)^{2}} e^{i \lambda_{h}^{n m} t}-\frac{\sqrt{2} n \pi}{\lambda^{n m}} \frac{1}{\left(\lambda^{n m}\right)^{2}} e^{i \lambda^{n m} t}\right|^{2} \leq \frac{\varepsilon}{8 c} .
$$

From (6.11) and (6.12), (6.10) follows immediately and the proof ends.

\subsection{Identification of the limit control}

In this section we show that the limit $(v, w)$ of the sequence $\left(v_{h}, z_{h}\right)_{h>0}$ from Theorem 6.2 is the HUM control for the continuous equation (1.2).

Theorem 6.3 We have that $(v, z)=\left(\widehat{w}_{x}(t, 1, y), \widehat{w}_{y}(t, x, 1)\right)$ is the HUM control for (1. Q) , where $\left(\widehat{w}, \widehat{w}^{\prime}\right)$ is the solution of (2. Q) with initial data $\left(\widehat{w}^{0}, \widehat{w}^{1}\right)$ given by (6.6).

Proof: By taking into account Proposition 2.1, the proof consists of verifying (2.8). 
The optimality condition for the minimizer of $\mathcal{J}_{h}$ provides the following characterization of $v_{h}$ and $z_{h}$

$$
\begin{gathered}
<\left(-K_{h}^{-1} M_{h} U_{h}^{1}, U_{h}^{0}\right),\left(W_{h}(0), W_{h}^{\prime}(0)\right)>_{0}= \\
\frac{h^{2}}{16} \int_{0}^{T}\left(\sum_{j=1}^{N}\left(2 v_{h, j}^{\prime}+v_{h, j+1}^{\prime}+v_{h, j-1}^{\prime}\right) w_{N j}^{\prime}+\sum_{i=1}^{N}\left(2 z_{h, i}^{\prime}+z_{h, i+1}^{\prime}+z_{h, i-1}^{\prime}\right) w_{i N}^{\prime}\right) d t+ \\
+\frac{1}{3} \int_{0}^{T}\left(\sum_{j=1}^{N}\left(v_{h, j}+v_{h, j+1}+v_{h, j-1}\right) w_{N j}+\sum_{i=1}^{N}\left(z_{h, i}+z_{h, i+1}+z_{h, i-1}\right) w_{i N}\right) d t=0,
\end{gathered}
$$

for any $\left(W_{h}^{0}, W_{h}^{1}\right) \in \mathbb{R}^{2 N^{2}}$, where $\left(W_{h}, W_{h}^{\prime}\right)$ is the corresponding solution of (3.6).

Now we evaluate $(6.13)$ for $\left(W_{h}^{0}, W_{h}^{1}\right)=\Psi_{h}^{n m}$. We obtain that, for any $(n, m) \in \mathcal{I}_{N}$,

$$
\begin{aligned}
& \frac{\cos \left(\frac{n \pi h}{2}\right) \cos \left(\frac{m \pi h}{2}\right)}{\sqrt{2}}<\left(-K_{h}^{-1} M_{h} U_{h}^{1}, U_{h}^{0}\right), \Psi_{h}^{m n} e^{i \lambda_{h}^{m n} T}>_{0} \\
& =\int_{0}^{T} e^{i \lambda_{h}^{n m}(t-T)}\left[(-1)^{n+1} \sin (n \pi h)<C_{h} v_{h}^{\prime}, \phi_{h}^{m}>+(-1)^{m+1} \sin (m \pi h)<C_{h} z_{h}^{\prime}, \phi_{h}^{n}>\right] d t \\
& +\int_{0}^{T} \frac{e^{i \lambda_{h}^{n m}(t-T)}}{i \lambda_{h}^{n m}}\left[(-1)^{n+1} \sin (n \pi h)<B_{h} v_{h}, \phi_{h}^{m}>+(-1)^{m+1} \sin (m \pi h)<B_{h} z_{h}, \phi_{h}^{n}>\right] d t,
\end{aligned}
$$

which is equivalent to

$$
\begin{aligned}
i \cos \left(\frac{n \pi h}{2}\right) \cos \left(\frac{m \pi h}{2}\right) & <\left(-K_{h}^{-1} M_{h} U_{h}^{1}, U_{h}^{0}\right), \Psi_{h}^{m n}>_{0} \\
& =\frac{\sqrt{2} h^{2} i}{4} \int_{0}^{T} e^{i \lambda_{h}^{n m} t}\left[(-1)^{n+1} \sin (n \pi h) \cos ^{2}\left(\frac{m \pi h}{2}\right)\left(v_{h}^{\prime}, \phi_{h}^{m}\right)\right. \\
& \left.+(-1)^{m+1} \sin (m \pi h) \cos ^{2}\left(\frac{n \pi h}{2}\right)\left(z_{h}^{\prime}, \phi_{h}^{n}\right)\right] d t \\
& -\frac{\sqrt{2}}{3 \lambda_{h}^{n m}} \int_{0}^{T} e^{i \lambda_{h}^{n m} t}\left[(-1)^{n+1} \sin (n \pi h)(1+2 \cos (m \pi h))\left(v_{h}, \phi_{h}^{m}\right)\right. \\
& \left.+(-1)^{m+1} \sin (m \pi h)(1+2 \cos (n \pi h))\left(z_{h}, \phi_{h}^{n}\right)\right] d t .
\end{aligned}
$$

We have that

$$
<\left(-K_{h}^{-1} M_{h} U_{h}^{1}, U_{h}^{0}\right), \Psi_{h}^{m n}>_{0}=\frac{1}{i \lambda_{h}^{n m}} \alpha_{n m}^{h}, \quad<v_{h}, \phi_{h}^{m}>=\frac{1}{2 h} b_{h}^{m}(t), \quad<z_{h}, \phi_{h}^{n}>=\frac{1}{2 h} d_{h}^{n}(t) .
$$

By taking into account that, for every fixed $(n, m) \in \mathcal{I}_{N}$, when $h$ tends to zero we have that

$$
\begin{gathered}
\alpha_{n m}^{h} \rightarrow \alpha_{n m}, \quad \lambda_{h}^{n m} \rightarrow \lambda^{n m}, \\
b_{m}^{h}(t) \rightarrow b_{m}(t), \quad d_{m}^{h}(t) \rightarrow d_{m}(t) \text { in } L^{2}(0, T), \\
h\left(b_{m}^{h}\right)^{\prime}(t) \rightarrow 0, \quad h\left(d_{m}^{h}\right)^{\prime}(t) \rightarrow 0 \text { in } L^{2}(0, T),
\end{gathered}
$$

and by passing to the limit in (6.14) we obtain $(2.8)$. 


\section{Numerical experiments}

This section is devoted to present numerical experiments which illustrate the efficiency of scheme (3.4) in controllability problems. This is done by using a fully-discrete approximation derived from the semi-discrete scheme (3.4). In the first subsection we present the method and in the second one we consider two examples with different non smooth initial data and location of controls.

\subsection{Description of a fully discrete approximation}

We first introduce a fully discrete - in space and time - approximation method associated to system (2.2). This is precisely a classical time discretization of the semi-discrete scheme (3.4).

Given a time interval $[0, T]$ we introduce a uniform mesh $\left\{t^{k}=k \Delta t\right\}_{k=0, \ldots, M}$ with time-step $\Delta t$ and $T=M \Delta t$. Let us denote by $w_{i j}^{k}$ the approximation of the solution $w$ of $(2.2)$ at the point of coordinates $\left(x_{i}, y_{j}\right)$ and at time $t^{k}=k \Delta t$, i.e. $w_{i j}^{k} \approx w\left(k \Delta t, x_{i}, y_{j}\right)$.

A fully-discrete scheme may be obtained by replacing the time derivative $w_{i j}^{\prime \prime}\left(t^{k}\right)$ by the finite difference $\left(w_{i j}^{k+1}-2 w_{i j}^{k}+w_{i j}^{k-1}\right) / \Delta t^{2}$. If $W^{k}=\left(w_{i j}^{k}\right)_{1 \leq i, j \leq N} \in \mathbb{R}^{N^{2}}$, for $0 \leq k \leq M$, the vectorial form (3.6) becomes

$$
\left\{\begin{array}{l}
M_{h} \frac{W^{k+1}-2 W^{k}+W^{k-1}}{\Delta t^{2}}+K_{h} W^{k}=0, \quad 0 \leq k \leq M, \\
W^{M}=w^{0}, \frac{W^{M+1}-W^{M-1}}{2 \Delta t}=w^{1} .
\end{array}\right.
$$

The scheme (7.1) is consistent of order 2 in time and space with the continuous system (2.2) and it is stable under the so-called Courant-Friedrichs-Lewy (CFL) condition (see [3])

$$
\frac{\Delta t^{2}}{4} \sup _{W \in \mathbb{R}^{N^{2}}, W \neq 0} \frac{\left(K_{h} W, W\right)}{\left(M_{h} W, W\right)}<1, \quad \forall h, \Delta t>0 .
$$

Moreover, the discrete spectrum $\left(\lambda_{h, \Delta t}^{m n}\right)_{1 \leq m, n \leq N}$ associated to this scheme is

$$
\lambda_{h, \Delta t}^{m n}=\frac{2}{\Delta t} \arcsin \left(\frac{\Delta t}{2} \lambda_{h}^{m n}\right), \quad 1 \leq m, n \leq N
$$

with $\lambda_{h}^{m n}$ defined by (5.3). Therefore (7.2) implies the following condition:

$$
\Delta t \leq C h^{3}
$$

for some $C>0$ independent of $h$.

In order to relax this restrictive stability condition we use an implicit method replacing the term $K_{h} W^{k}$ in (7.1) by $1 / 4 K_{h}\left(W^{k+1}+2 W^{k}+W^{k-1}\right)$. Note that this corresponds to one of 
the Newmark methods (with parameters $\gamma=1 / 2$ and $\beta=1 / 4, \quad$ ). Thus, we obtain the following scheme

$$
\left\{\begin{array}{c}
\left(M_{h}+\frac{\Delta t^{2}}{4} K_{h}\right) \frac{W^{k+1}-2 W^{k}+W^{k-1}}{\Delta t^{2}}+K_{h} W^{k}=0, \quad 0 \leq k \leq M \\
W^{M}=w^{0}, \frac{W^{M+1}-W^{M-1}}{2 \Delta t}=w^{1}
\end{array}\right.
$$

consistent with the continuous system (2.2) and unconditionally stable for any value of $\Delta t$.

Let us now analyze if this fully-discrete system conserves the observability properties of the semi-discrete scheme. Following the analysis in Section 4 we study the group velocity of discrete plane waves of the form

$$
w_{i j}^{k}=e^{i\left(\xi \cdot\left(x_{i}, x_{j}\right)-\omega t^{k}\right)}, \quad \xi=\left(\xi_{1}, \xi_{2}\right)
$$

For the discrete system (7.4) the following relation between modes $\xi$ and frequencies $\omega$ holds

$$
\omega(\xi)=\frac{2}{\Delta t} \arcsin \left(\frac{\Delta t}{2} \sqrt{\frac{\omega_{s}(\xi)^{2}}{1+\frac{\Delta t^{2}}{4} \omega_{s}(\xi)^{2}}}\right),
$$

where

$$
\omega_{s}(\xi)=\frac{2}{h} \sqrt{\tan ^{2}\left(\frac{\xi_{1} h}{2}\right)+\tan ^{2}\left(\frac{\xi_{2} h}{2}\right)+\frac{2}{3} \tan ^{2}\left(\frac{\xi_{1} h}{2}\right) \tan ^{2}\left(\frac{\xi_{2} h}{2}\right)}
$$

and $\xi \in[-\pi / h, \pi / h]^{2}$.

The group velocity associated to a mode $\xi$ in a direction $v=\left(v_{1}, v_{2}\right)$ is given by $\nabla_{\xi} \omega \cdot v$ and a necessary condition in order to have a uniform (in $h$ and $\Delta t$ ) observability property in finite time is to have a uniform bound from below (in $\xi, h$ and $\Delta t$ ) for $\left|\nabla_{\xi} \omega\right|=\sqrt{\left|\partial_{\xi_{1}} \omega\right|^{2}+\left.\partial_{\xi_{2}} \omega\right|^{2}}$, i.e.

$$
\left|\nabla_{\xi} \omega\right| \geq C>0, \text { for all } \xi, h \text { and } \Delta t
$$

A straightforward computation shows that the minimum value of $\left|\nabla_{\xi} \omega\right|$ is obtained for $\xi=$ $(\pi / h, \pi / h)$ and that

$$
\left|\nabla_{\xi} \omega(\pi / h, \pi / h)\right| \sim h^{3 / 2} \Delta t^{-1}
$$

Therefore, this is uniformly bounded from below if

$$
\Delta t=C h^{3 / 2}, \quad \forall C>0
$$

Thus, even if the scheme (7.4) is stable for any discretization step $\Delta t$, in order to guarantee a uniform (in $h$ and $\Delta t$ ) controllable scheme, (7.6) should be verified.

Note that the implicit method (7.4)-(7.6) permits to gain a factor $h^{3 / 2}$ in the ratio $\Delta t / h$ compared with the initial scheme (7.1) for which stability is ensured by (7.3). 


\subsection{Numerical examples}

In this section, we present some numerical experiments for two different initial conditions. The first example is a well-known test proposed by Glowinski-Li-Lions in [6] for which the initial velocity $u^{1}$ is discontinuous. The second example is even more singular, involving a discontinuous initial displacement $u^{0}$. Each one of these examples is defined in the unit square.

The HUM control is obtained by minimizing the functional $\mathcal{J}$ in (5.1) and then by using (5.2). Following [6], the iterative conjugate gradient algorithm is used with the initialization $\left(\hat{W}_{h}^{0}, \hat{W}_{h}^{1}\right)=(0,0)$. We assume that the convergence is obtained when the corresponding relative residual is lower or equal than $e=10^{-8}$.

\subsubsection{Example 1: Discontinuity of the initial velocity $u^{1}$}

Firstly, we consider the Glowinski-Li-Lions example . The initial data to be controlled, $\left(u^{0}, u^{1}\right)$, is constituted by a Lipschitz continuous function $u^{0}$ not belonging to $C^{1}(\bar{\Omega})$ and a function $u^{1}$ belonging to $L^{\infty}(\Omega)$ but not to $C^{0}(\bar{\Omega})$. The explicit expressions of $\left(u^{0}, u^{1}\right)$ may be found in [6]. The interest of this example is that the analytical solution is known. More precisely, let us consider $T=15 / 4 \sqrt{2}$ and the solution of the wave equation (2.2) given by

$$
\hat{u}(t, x, y)=\sqrt{2} \cos \left(\pi \sqrt{2}\left(T-t-\frac{1}{4 \sqrt{2}}\right)\right) \sin (\pi x) \sin (\pi y)
$$

Let $\left(\hat{u}^{0}, \hat{u}^{1}\right)$ be its corresponding initial data. Then $V=\left.\frac{\partial \hat{u}}{\partial \nu}\right|_{2}$ is exactly the HUM control acting on the whole boundary $\partial \Omega$ which leads $\left(u^{0}, u^{1}\right)$ to the rest in time $T$.

In [6] the simplest discretization for the wave equation is considered. It consists in the fivepoints formula in space for the Laplacian, combined with the usual three-points formula for the second derivative in time. This produces an explicit scheme for which condition (7.5) fails. The conjugate gradient algorithm based on this scheme diverges. Several cures have been proposed to obtain convergence without changing the scheme, such as filtering with a bi-grid strategy or a Tychonoff regularization technique

Table 1 displays the good behavior of the scheme (7.4) when $h$ and $\Delta t=h^{3 / 2}$ are decreasing, by comparing the exact and approximate results for the initial data giving the control and for the control itself. $\left|\hat{w}^{0}\right|_{H^{1}(\Omega)}$ is defined by $\left|\dot{w}^{0}\right|_{H^{1}(\Omega)}=\left(\int_{\Omega}\left|\nabla \dot{w}^{0}\right|^{2} d x d y\right)^{1 / 2}$ whereas the $H^{-1}$ norm of $u^{1}$ in $\Omega$ is defined by $\|\left. u^{1}\right|_{H^{-1}}=|w|_{H^{1}(\Omega)}$ where $w \in H_{0}^{1}(\Omega)$ is the solution of the Dirichlet problem $-\Delta w=u^{1}$ in $\Omega, w=0$ on $\Gamma$. 
Remark 7.1 An analysis of the results from Table 1 shows that the number of conjugate gradient iterations necessary to achieve convergence is independent of $h$. Moreover, the approximation errors for $\left(\dot{w}^{0}, \dot{w}^{1}\right)$ satisfy

$$
\left\|\dot{w}^{0}-\hat{W}_{h}^{0}\right\|_{L^{2}(\Omega)}=O\left(h^{1.88}\right), \quad\left\|\dot{w}^{0}-\hat{W}_{h}^{0}\right\|_{H_{0}^{1}(\Omega)}=O\left(h^{1.10}\right), \quad\left\|\dot{w}^{1}-\hat{W}_{h}^{1}\right\|_{H^{1}(\Omega)}=O\left(h^{1.06}\right)
$$

while for the control, we have

$$
\left\|V-V_{h}\right\|_{L^{2}(\alpha \Omega \times(0, T))}=O\left(h^{1.17}\right)
$$

Figure 2-left depicts the exact and approximate controls $V$ and $V_{h}$ at the point $\bar{x}=(1,1 / 2) \in \partial \Omega$, obtained with $h=1 / 15$ (for $h=1 / 30,1 / 60,1 / 120$, the two curves can not be distinguished). The approximation error is given in figure 2-right and satisfies $\left\|\left(V-V_{h}\right)\right\|_{L^{\infty}(0, T)}=O\left(h^{0.95}\right)$.

At last, some numerical experiments (not reproduced here) highlight the condition (7.6). More precisely, if the unconditionally stable scheme (7.4) is used with $\Delta t=O(h)$, then the conjugate gradient algorithm diverges for $h$ small enough.

\begin{tabular}{c|cccc}
\hline & $h=1 / 15$ & $h=1 / 30$ & $h=1 / 60$ & $h=1 / 120$ \\
\hline $\begin{array}{c}\text { CG iterations } \\
\frac{\left\|\hat{w}^{0}-\hat{W}_{h}^{0}\right\|_{L^{2}(\Omega)}}{\left\|\hat{w}^{0}\right\|_{L^{2}(\Omega)}}\end{array}$ & 5 & 6 & 6 & 6 \\
$\frac{\left|\hat{\omega}^{0}-\hat{W}_{h}^{0}\right|_{H^{1}(\Omega)}}{\left|\hat{w}^{0}\right|_{H^{1}(\Omega)}}$ & $4.01 \times 10^{-2}$ & $5.53 \times 10^{-3}$ & $1.43 \times 10^{-3}$ & $5.27 \times 10^{-4}$ \\
$\frac{\left\|\hat{w}^{1}-\hat{W}_{h}^{1}\right\|_{L^{2}(\Omega)}}{\left\|\hat{w}^{1}\right\|_{L^{2}(\Omega)}}$ & $4.45 \times 10^{-2}$ & $2.13 \times 10^{-2}$ & $9.64 \times 10^{-3}$ & $4.86 \times 10^{-3}$ \\
$\frac{\left\|V-V_{h}\right\|_{L^{2}(\sigma \Omega \times(0, T))}}{\|V\|_{L^{2}(\sigma \Omega \Omega(0, T))}}$ & $2.31 \times 10^{-1}$ & $1.24 \times 10^{-1}$ & $4.93 \times 10^{-2}$ & $2.08 \times 10^{-2}$ \\
$\left\|V_{h}\right\|_{L^{2}(\partial \Omega \times(0, T))}$ & 7.4187 & 7.3782 & 7.3812 & 7.3859 \\
$E_{h}(T) / E_{h}(0)$ & $1.55 \times 10^{-3}$ & $4.1 \times 10^{-4}$ & $5.61 \times 10^{-5}$ & $1.01 \times 10^{-6}$ \\
\hline
\end{tabular}

Table 1: Results obtained with $\Delta t=h^{3 / 2}$ in Example 1. The control is active on $\partial \Omega$ and $T=15 / 4 \sqrt{2}$. 

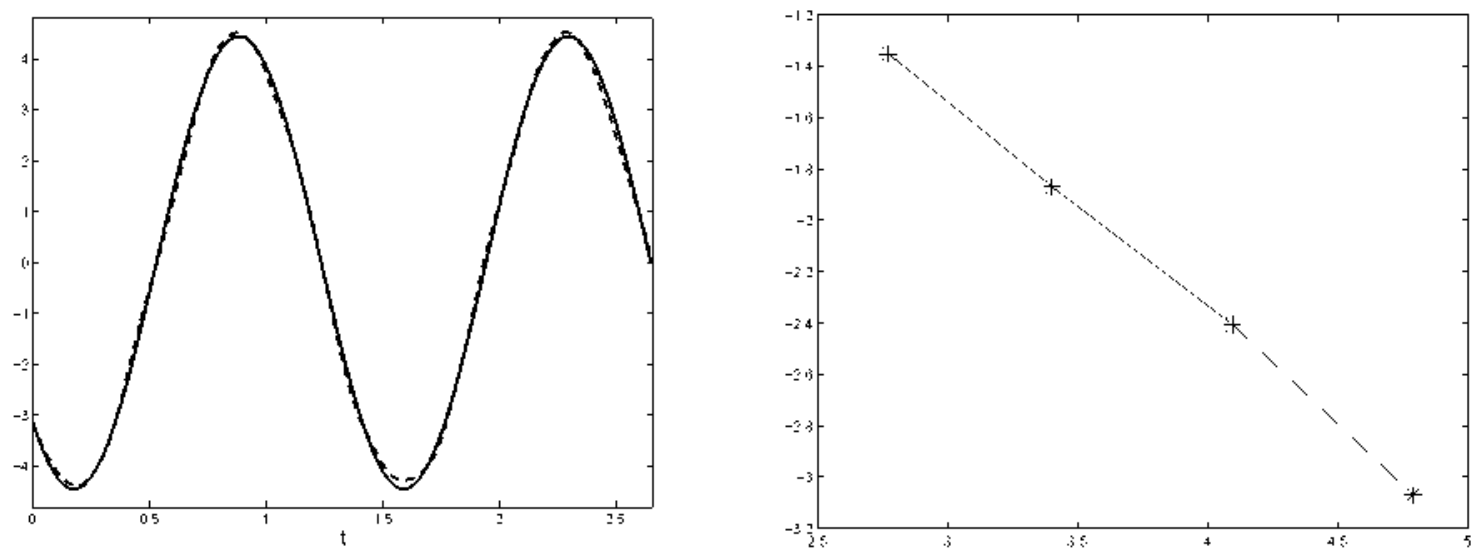

Figure 2: Left: Exact control $V(t, \bar{x})$ (dashed line) and approximate control $V_{h}$ (solid line) at the point $\bar{x}=(x, y)=(1,1 / 2) \in \partial \Omega$ with $h=1 / 15$; Right : $\log \left(\left\|\left(V-V_{h}\right)(\cdot, \bar{x})\right\|_{\left.L_{(0, T)}^{\infty}\right)}\right.$ vs. $\log (1 / h)$ (Example 1).

\subsubsection{Example 2: Discontinuity of the initial position $u^{0}$}

In this second example, we consider a more singular situation with a discontinuous initial displacement $u^{0}$ :

$$
u^{0}(x, y)=\left\{\begin{array}{ll}
40 & (x, y) \in\left(\frac{1}{3}, \frac{2}{3}\right)^{2} \\
0 & \text { elsewhere }
\end{array} \quad ; \quad u^{1}(x, y)=0 .\right.
$$

We assume that the control $(v, z)$ is active on $\Gamma_{1}$ (see (1.1)) and we take $T=2 \sqrt{2}$. As in the previous example, a conjugate gradient algorithm based on the simplest discretization of the wave equation diverges. On the contrary, the use of scheme (7.4) allows to obtain convergence without filtering or regularization techniques. This is displayed in Table 2. The number of iterations to achieve convergence remains low and constant for $h$ small. Moreover, the convergence is slightly affected by the lack of regularity of $u^{0}$ : we compute for instance that $\left\|u^{1}-U_{h}^{1}\right\|_{H^{-1}(\Omega)}=O\left(h^{0.71}\right)$ to be compared with $\left\|u^{1}-U_{h}^{1}\right\|_{H^{-1}(\Omega)}=O\left(h^{1.01}\right)$ for the first example.

For $h=1 / 60$, the exact controllability of the wave equation is illustrated on Figure 3: the approximate controlled solution $U_{h}$ is drawn in the unit square $\Omega$ for six values of time: $t=0, T / 5,2 T / 5,3 T / 5,4 T / 5$ and $T$. For $t=0, U_{h}$ coincides with the discontimuous position $u^{0}$ while for $t=T$ the solution is null controlled: the ratio of the energy between the two states is $E_{h}(T) / E_{h}(0) \approx 1.11 \times 10^{-4}$. At last, we highlight that the value of $T$ is strictly lower than $2 \sqrt{3}$ obtained in Theorem 4.1 . 

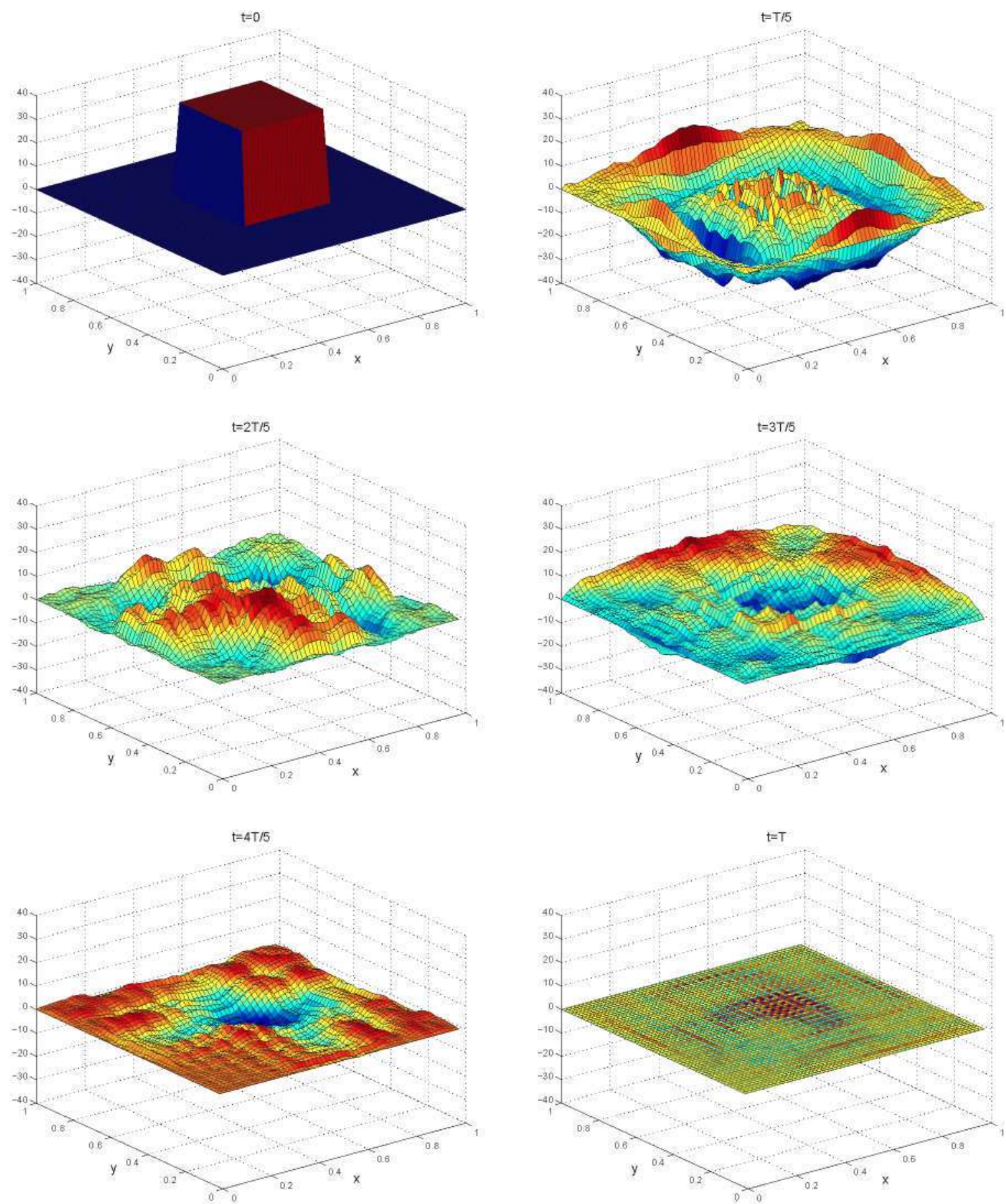

Figure 3: Controllability of the initial data (7.7) in $\Omega=(0,1)^{2}$ : approximations $U_{h}(t)$ of the controlled solution for $t=0, T / 5,2 T / 5,3 T / 5,4 T / 5$ and $T=2 \sqrt{2}$ with $h=1 / 60$ in Example 2 . 


\begin{tabular}{c|cccc}
\hline & $h=1 / 15$ & $h=1 / 30$ & $h=1 / 60$ & $h=1 / 120$ \\
\hline CG iterations & 13 & 11 & 10 & 10 \\
$\left\|\hat{W}_{h}^{0}\right\|_{L^{2}(\Omega)}$ & $1.50 \times 10^{-1}$ & $1.35 \times 10^{-1}$ & $1.31 \times 10^{-1}$ & $1.30 \times 10^{-4}$ \\
$\left|\hat{W}_{h}^{0}\right|_{H^{1}(\Omega)}$ & 1.0990 & 1.1071 & 1.1147 & 1.1169 \\
$\left\|\dot{W}_{h}^{1}\right\|_{L^{2}(\Omega)}$ & 5.871 & 5.425 & 5.196 & 5.164 \\
$\left\|v_{h}\right\|_{L^{2}((0,1) \times(0, T))}+\left\|z_{h}\right\|_{L^{2}((0,1) \times(0, T))}$ & $1.290 \times 10^{1}$ & $1.243 \times 10^{1}$ & $1.222 \times 10^{1}$ & $1.218 \times 10^{1}$ \\
$E_{h}(T) / E_{h}(0)$ & $4.30 \times 10^{-3}$ & $3.68 \times 10^{-4}$ & $1.11 \times 10^{-4}$ & $8.39 \times 10^{-5}$ \\
\hline
\end{tabular}

Table 2: Results obtained with $\Delta t=h^{3 / 2}$ in Example 2. The control is active on $\Gamma_{1} \subset \partial \Omega$ and $T=2 \sqrt{2}$. The last row indicates that the system is controlled at time $T$.

Furthermore, a very useful result to validate our numerical scheme for large values of $T$ is due to Bensoussan that, when the control is active on the whole boundary,

$$
\lim _{T \rightarrow \infty} T\left(\hat{u}_{T}^{0}, \hat{u}_{T}^{1}\right)=\left(\chi^{0}, \chi^{1}\right)
$$

where $\chi^{0}$ and $\chi^{1}$ are solution of

$$
\Delta \chi^{0}=\frac{1}{2} u^{1} \text { in } \Omega, \quad \chi^{0}=0 \text { on } \partial \Omega ; \quad \chi^{1}=\frac{1}{2} u^{0} \text { in } \bar{\Omega} .
$$

and $\left(\dot{w}_{T}^{0}, w_{T}^{1}\right)$ are the initial conditions of the backward system $(2.2)$. The numerical results we obtain with the scheme (7.4) (see Table 3 ) confirms clearly the theoretical property (7.8): $\left\|T \dot{W}_{T h}^{0}-\chi_{h}^{0}\right\|_{H_{0}^{1}(\Omega)}=O\left(T^{-1.10}\right)$ and $\left\|T \dot{W}_{T h}^{1}-\chi_{h}^{1}\right\|_{L^{2}(\Omega)}=O\left(T^{-1.0087}\right)$. As advocated in [6], these results provide a validation of the numerical methodology introduced here and show that the scheme is particularly robust, accurate and perfectly able to handle very long intervals $[0, T]$.

\begin{tabular}{c|ccccc}
\hline & $T=3$ & $T=5$ & $T=10$ & $T=20$ & $T=40$ \\
\hline CG iterations & 10 & 9 & 8 & 8 & 5 \\
$\left\|T \hat{W}_{T h}^{0}-\chi_{h}^{0}\right\|_{H_{0}^{1}(\Omega)}$ & $7.15 \times 10^{-1}$ & $3.4 \times 10^{-1}$ & $1.40 \times 10^{-1}$ & $1.11 \times 10^{-1}$ & $3.3 \times 10^{-2}$ \\
$\left\|T \hat{W}_{T h}^{1}-\chi_{h}^{1}\right\|_{L^{2}(\Omega)}$ & $4.12 \times 10^{-1}$ & $2.21 \times 10^{-1}$ & $1.55 \times 10^{-1}$ & $8.46 \times 10^{-2}$ & $2.47 \times 10^{-2}$ \\
\hline
\end{tabular}

Table 3: $\left\|T \dot{W}_{T h}^{0}-\chi_{h}^{0}\right\|_{H_{0}^{1}(\Omega)}$ and $\left\|T \dot{W}_{T h}^{1}-\chi_{h}^{1}\right\|_{L^{2}(\Omega)}$ with $h=1 / 60$ in Example 2 . 
The numerical results we have presented indicate that the scheme (7.4) under condition (7.6) provides a uniform approximation of the control, with respect to the discretization parameters. However, a rigorous proof of the convergence remains to be done.

\section{A Appendix}

In this section we prove Theorem 4.1. To simplify the notation we write

$$
\begin{aligned}
a_{i j}^{k l} & =w_{i k}+w_{i l}+w_{j k}+w_{j l}, \quad b_{i j}^{k l}=w_{i k}^{\prime}+w_{i l}^{\prime}+w_{j k}^{\prime}+w_{j l}^{\prime}, \\
c_{i j}^{k l} & =w_{i k}^{\prime \prime}+w_{i l}^{\prime \prime}+w_{j k}^{\prime \prime}+w_{j l}^{\prime \prime}, \\
\Delta_{(1,0)} w_{i j} & =2 w_{i j}-w_{i+1 j}-w_{i-1 j}, \quad \Delta_{(0,1)} w_{i j}=2 w_{i j}-w_{i j+1}-w_{i j-1}, \\
\Delta_{(1,1)} w_{i j} & =2 w_{i j}-w_{i+1 j+1}-w_{i-1 j-1}, \quad \Delta_{(1,-1)} w_{i j}=2 w_{i j}-w_{i+1 j-1}-w_{i-1 j+1} .
\end{aligned}
$$

When multiplying the discrete system by the discrete version of the usual continuous multiplier $(x, y) \cdot \nabla u$, i.e.

$$
(i h, j h) .\left(\frac{w_{i+1 j}-w_{i-1 j}}{2 h}, \frac{w_{i j+1}-w_{i-1 j}}{2 h}\right)=i \frac{w_{i+1 j}-w_{i-1 j}}{2}+j \frac{w_{i j+1}-w_{i j-1}}{2} \equiv \frac{m_{i j}}{2},
$$

and summing in $i$ and $j$ we obtain

$$
\begin{aligned}
0= & \frac{h^{2}}{32} \underbrace{\int_{0}^{T} \sum_{i, j=1}^{N}\left(c_{i i+1}^{j j+1}+c_{i i+1}^{j-1 j}+c_{i-1 i}^{j j+1}+c_{i-1 i}^{j-1 j}\right) m_{i, j} d t}_{\equiv C} \\
& +\frac{1}{6} \int_{0}^{T} \underbrace{\sum_{i, j=1}^{N}\left(\Delta_{(1,0)} w_{i j}+\Delta_{(0,1)} w_{i j}+\Delta_{(1,1)} w_{i j}+\Delta_{(1,-1)} w_{i j}\right) m_{i j}}_{\equiv D} d t
\end{aligned}
$$

We study separately $C$ and $D$. Integration by parts in $C$ allows us to obtain,

$$
C=\int_{0}^{T} C_{1} d t+\left[C_{2}\right]_{0}^{T}
$$

where

$$
\begin{aligned}
& C_{1}=-\sum_{i, j=1}^{N}\left(b_{i i+1}^{j j+1}+b_{i i+1}^{j-1 j}+b_{i-1 i}^{j j+1}+b_{i-1 i}^{j-1 j}\right) m_{i, j}^{\prime}, \\
& C_{2}=\sum_{i, j=1}^{N}\left(b_{i i+1}^{j j+1}+b_{i i+1}^{j-1 j}+b_{i-1 i}^{j j+1}+b_{i-1 i}^{j-1 j}\right) m_{i j} .
\end{aligned}
$$


We first consider the term $C_{1}$ above. In order to have the common factor $b_{i i+1}^{j j+1}$, we change the indexes in the last three terms of $C_{1}$ above. Then, taking into account that $w_{i, 0}=w_{i, N+1}=$ $w_{0, j}=w_{j, N+1}=0$ and after simplification, we obtain

$$
C_{1}=2 \sum_{i, j=0}^{N}\left(b_{i i+1}^{j j+1}\right)^{2}-(N+1)\left[\sum_{i=1}^{N}\left(w_{i N}^{\prime}+w_{i+1 N}^{\prime}\right)^{2}+\sum_{j=1}^{N}\left(w_{N j}^{\prime}+w_{N j+1}^{\prime}\right)^{2}\right] .
$$

We now analyze the term $D$ in (A.1). We only make the details for the first term in $D$ since the others can be simplified similarly. It reads,

$$
\sum_{i, j=1}^{N} \Delta_{(1,0)} w_{i j} m_{i j}=\sum_{i, j=1}^{N} \Delta_{(1,0)} w_{i j}\left[i\left(w_{i+1 j}-w_{i-1 j}\right)+j\left(w_{i j+1}-w_{i j-1}\right)\right] .
$$

We consider separately these two terms. For the second one we have

$$
\begin{gathered}
\Delta_{(1,0)} w_{i j} j\left(w_{i j+1}-w_{i j-1}\right)=\sum_{i, j=1}^{N} j\left(w_{i j}-w_{i-1 j}\right) w_{i j+1}-\sum_{i, j=1}^{N} j\left(w_{i+1 j}-w_{i j}\right) w_{i j+1} \\
-\left[\sum_{i, j=1}^{N} j\left(w_{i j}-w_{i-1 j}\right) w_{i j-1}-\sum_{i, j=1}^{N} j\left(w_{i+1 j}-w_{i j}\right) w_{i j-1}\right] .
\end{gathered}
$$

Changing the indexes to obtain the common factor $\left(w_{i+1 j}-w_{i j}\right)$ in all the terms and taking into account that $w_{i, 0}=w_{i, N+1}=w_{0, j}=w_{j, N+1}=0$, we obtain

$$
\begin{aligned}
& \sum_{i, j=0}^{N}\left[j\left(w_{i+1 j}-w_{i j}\right)\left(w_{i+1 j+1}-w_{i j+1}\right)-j\left(w_{i+1 j}-w_{i j}\right)\left(w_{i+1 j-1}-w_{i j-1}\right)\right] \\
& \quad=-\sum_{i, j=0}^{N}\left(w_{i+1 j+1}-w_{i j+1}\right)\left(w_{i+1 j}-w_{i j}\right) .
\end{aligned}
$$

An analogous argument allows to simplify the first term in (A.6) and the other three terms in $D$. We finally have

$$
\begin{aligned}
D & =-\sum_{i, j=0}^{N}\left[\left(w_{i+1 j+1}-w_{i+1 j}\right)\left(w_{i j+1}-w_{i j}\right)+\left(w_{i+1 j+1}-w_{i j+1}\right)\left(w_{i+1 j}-w_{i j}\right)\right] \\
& +\sum_{i, j=0}^{N}\left[\left(w_{i+1 j}-w_{i j}\right)^{2}+\left(w_{i j+1}-w_{i j}\right)^{2}\right]-(N+1) \sum_{j=0}^{N}\left[\left(w_{N j}\right)^{2}+2 w_{N j} w_{N j+1}\right] \\
& -(N+1) \sum_{i=0}^{N}\left[\left(w_{i N}\right)^{2}+2 w_{i N} w_{i+1 N}\right] .
\end{aligned}
$$


By Young's inequality we can estimate the first term in this formula,

$$
\begin{aligned}
& \sum_{i, j=0}^{N}\left[\left(w_{i+1 j+1}-w_{i+1 j}\right)\left(w_{i j+1}-w_{i j}\right)+\left(w_{i+1 j+1}-w_{i j+1}\right)\left(w_{i+1 j}-w_{i j}\right)\right] \\
& \quad \leq \sum_{i, j=0}^{N}\left[\left(w_{i j+1}-w_{i j}\right)^{2}+\left(w_{i+1 j}-w_{i j}\right)^{2}\right] .
\end{aligned}
$$

Therefore,

$$
\begin{aligned}
D & \geq-(N+1)\left[\sum_{j=0}^{N} w_{N j}^{2}+2 \sum_{j=0}^{N} w_{N j} w_{N j+1}+\sum_{i=0}^{N} w_{i N}^{2}+2 \sum_{i=0}^{N} w_{i N} w_{i+1 N}\right] \\
& =-(N+1)\left[\sum_{j=1}^{N}\left(w_{N j-1}+w_{N j}+w_{N j+1}\right) w_{N j}+\sum_{i=1}^{N}\left(w_{i-1 N}+w_{i N}+w_{i+1 N}\right) w_{i N}\right] .
\end{aligned}
$$

Substituting (A.2), (A.5) and (A.8) into (A.1) we obtain

$$
\begin{aligned}
& h^{2} \int_{0}^{T} \sum_{i, j=0}^{N}\left(\frac{b_{i i+1}^{j j+1}}{4}\right)^{2} d t \leq \frac{h}{8} \int_{0}^{T}\left[\sum_{i=1}^{N}\left(\frac{w_{i N}^{\prime}+w_{i+1 N}^{\prime}}{2}\right)^{2}+\sum_{j=1}^{N}\left(\frac{w_{N j}^{\prime}+w_{N j+1}^{\prime}}{2}\right)^{2}\right] d t \\
& +\frac{1}{2} \int_{0}^{T}\left[\sum_{j=1}^{N} \frac{w_{N j-1}+w_{N j}+w_{N j+1}}{3} \frac{w_{N j}}{h}+\sum_{i=1}^{N} \frac{w_{i-1 N}+w_{i N}+w_{i+1 N}}{3} \frac{w_{i N}}{h}\right] d t \\
& -\frac{h^{2}}{32}\left[C_{2}\right]_{0}^{T} .
\end{aligned}
$$

We observe that the term in the left hand side contains only one part of the energy. In order to obtain the full energy we make an equipartition of the energy. The following lemma is a discrete version of the well-known equipartition of energy for the continuous wave equation, which reads

$$
\left.0=-\int_{0}^{T} \int_{\Omega}\left(\left|w_{t}\right|^{2}+|\nabla u|^{2}\right) d x d t+\int_{\Omega}\left|w_{t} u\right|^{2} d x\right]_{0}^{T} .
$$

Lemma A.1 The following holds:

$$
\begin{aligned}
0= & -h^{2} \int_{0}^{T}\left[\sum_{i, j=0}^{N}\left(\frac{b_{i i+1}^{j j+1}}{4}\right)^{2}\right] d t+h^{2}\left[\sum_{i, j=0}^{N}\left(\frac{a_{i i+1}^{j j+1}}{4}\right)\left(\frac{b_{i i+1}^{j j+1}}{4}\right)\right]_{0}^{T} \\
+ & h^{2} \sum_{i, j=0}^{N} \int_{0}^{T}\left[\frac{1}{3}\left(\frac{w_{i+1 j}-w_{i j}}{h}\right)^{2}+\frac{1}{3}\left(\frac{w_{i j+1}-w_{i j}}{h}\right)^{2}\right. \\
& \left.+\frac{2}{3}\left(\frac{w_{i+1 j+1}-w_{i j}}{h \sqrt{2}}\right)^{2}+\frac{2}{3}\left(\frac{w_{i+1 j}-w_{i j+1}}{h \sqrt{2}}\right)^{2}\right] d t
\end{aligned}
$$


The proof of this lemma is straightforward following the idea of the continuous system where (A.10) is obtained multiplying system (2.2) by $u$ and integrating by parts.

When applying Lemma A.1 to the identity (A.9) we obtain

$$
\begin{aligned}
\int_{0}^{T} E_{h}(t) d t+\frac{h^{2}}{32}\left[\sum_{i, j=0}^{N} a_{i i+1}^{j j+1} b_{i i+1}^{j j+1}+C_{2}\right]_{0}^{T} \\
\leq \frac{h}{8} \int_{0}^{T}\left[\sum_{i=1}^{N}\left(\frac{w_{i N}^{\prime}+w_{i+1 N}^{\prime}}{2}\right)^{2}+\sum_{j=1}^{N}\left(\frac{w_{N j}^{\prime}+w_{N j+1}^{\prime}}{2}\right)^{2}\right] d t \\
\quad+\frac{1}{2} \int_{0}^{T}\left[\sum_{j=1}^{N} \frac{w_{N j-1}+w_{N j}+w_{N j+1}}{3} \frac{w_{N j}}{h}+\sum_{i=1}^{N} \frac{w_{i-1 N}+w_{i N}+w_{i+1 N}}{3} \frac{w_{i N}}{h}\right]
\end{aligned}
$$

The following lemma allows us to estimate the the second term in the left hand side of this formula.

Lemma A.2 The following holds

$$
h^{2}\left[\sum_{i, j=0}^{N} a_{i i+1}^{j j+1} b_{i i+1}^{j j+1}+C_{2}\right]_{0}^{T} \leq 64 \sqrt{3} E_{h}(0) .
$$

Before proving this lemma we finish the proof of Theorem 4.1.

From Lemma A.2 and the conservation of the discrete energy, stated in Proposition 4.1, we have

$$
\int_{0}^{T} E_{h}(t) d t+\frac{h^{2}}{32}\left[\sum_{i, j=0}^{N} a_{i i+1}^{j j+1} b_{i i+1}^{j j+1}+C_{2}\right]_{0}^{T} \geq T E_{h}(0)-2 \sqrt{3} E_{h}(0)=(T-2 \sqrt{3}) E_{h}(0),
$$

which combined with (A.11) provides the following,

$$
\begin{aligned}
& (T-2 \sqrt{3}) E_{h}(0) \leq \frac{h}{8} \int_{0}^{T}\left[\sum_{i=1}^{N}\left(\frac{w_{i N}^{\prime}+w_{i+1 N}^{\prime}}{2}\right)^{2}+\sum_{j=1}^{N}\left(\frac{w_{N j}^{\prime}+w_{N j+1}^{\prime}}{2}\right)^{2}\right] d t \\
& +\frac{1}{2} \int_{0}^{T}\left[\sum_{j=1}^{N} \frac{w_{N j-1}+w_{N j}+w_{N j+1}}{3} \frac{w_{N j}}{h}+\sum_{i=1}^{N} \frac{w_{i-1 N}+w_{i N}+w_{i+1 N}}{3} \frac{w_{i N}}{h}\right] d t .
\end{aligned}
$$

This concludes the proof of Theorem 4.1.

Proof of Lemma A.2. From (A.4) we have

$$
\sum_{i, j=0}^{N} a_{i i+1}^{j j+1} b_{i i+1}^{j j+1}+C_{2}=\sum_{i, j=0}^{N} a_{i i+1}^{j j+1} b_{i i+1}^{j j+1}+\sum_{i, j=1}^{N}\left[b_{i i+1}^{j j+1}+b_{i i+1}^{j-1 j}+b_{i-1 i}^{j j+1}+b_{i-1 i}^{j-1 j}\right] m_{i j} .
$$


To simplify the notation we assume that $w_{N+2 j}=w_{N j}, w_{i N+2}=w_{i N}, w_{-1 j}=w_{i,-1}=0$, for all $i, j=0, \ldots, N+1$. We change the indexes in each one of the terms of the right hand side of (A.13) in order to have the common factor $b_{i i+1}^{j+1}$. Then we obtain

$$
\sum_{i, j=0}^{N} a_{i i+1}^{j j+1} b_{i i+1}^{j j+1}+C_{2}=\sum_{i, j=0}^{N}\left(a_{i i+1}^{j j+1}+R_{i j}\right) b_{i i+1}^{j j+1}
$$

where

$$
\begin{aligned}
& R_{i j}=i\left[\left(w_{i+1 j}-w_{i-1 j}\right)+\left(w_{i+1 j+1}-w_{i-1 j+1}\right)\right]+(i+1)\left[\left(w_{i+2 j}-w_{i j}\right)+\left(w_{i+2 j+1}-w_{i j+1}\right)\right] \\
& \quad+j\left[\left(w_{i j+1}-w_{i j-1}\right)+\left(w_{i+1 j+1}-w_{i+1 j-1}\right)\right]+(j+1)\left[\left(w_{i j+2}-w_{i j}\right)+\left(w_{i+1 j+2}-w_{i+1 j}\right)\right]
\end{aligned}
$$

We estimate the right hand side in (A.14) using the Schwartz inequality. Thus,

$$
\sum_{i, j=0}^{N} a_{i i+1}^{j j+1} b_{i i+1}^{j j+1}+C_{2} \leq\left[\sum_{i, j=0}^{N}\left(a_{i i+1}^{j j+1}+R_{i j}\right)^{2}\right]^{1 / 2}\left[\sum_{i, j=0}^{N}\left(b_{i i+1}^{j j+1}\right)^{2}\right]^{1 / 2}
$$

Now we prove that

$$
\sum_{i, j=0}^{N}\left(a_{i i+1}^{j j+1}+R_{i j}\right)^{2} \leq \sum_{i, j=0}^{N} R_{i j}^{2}+8 \sum_{i=1}^{N}(N+1)\left(w_{i N}\right)^{2}+8 \sum_{j=1}^{N}(N+1)\left(w_{N j}\right)^{2} .
$$

Indeed, we have

$$
\sum_{i, j=0}^{N}\left[\left(a_{i i+1}^{j j+1}+R_{i j}\right)^{2}-R_{i j}^{2}\right]=\sum_{i, j=0}^{N}\left[\left(a_{i i+1}^{j j+1}\right)^{2}+2 a_{i i+1}^{j j+1} R_{i j}\right]
$$

and it is not difficult to see that

$$
\sum_{i, j=0}^{N} a_{i i+1}^{j j+1} R_{i j}=-2 \sum_{i, j=0}^{N}\left(a_{i i+1}^{j j+1}\right)^{2}+\sum_{i=1}^{N}(N+1)\left(w_{i N}+w_{i+1 N}\right)^{2}+\sum_{j=1}^{N}(N+1)\left(w_{N j}+w_{N j+1}\right)^{2}
$$

Therefore, using Young's inequality, the right hand side in (A.17) reads

$$
\begin{aligned}
& \sum_{i, j=0}^{N}\left[\left(a_{i i+1}^{j j+1}\right)^{2}+2 a_{i i+1}^{j j+1} R_{i j}\right]=-3 \sum_{i, j=0}^{N}\left(a_{i i+1}^{j j+1}\right)^{2}+2 \sum_{i=1}^{N}(N+1)\left(w_{i N}+u_{i+1 N}\right)^{2} \\
& +2 \sum_{j=1}^{N}(N+1)\left(w_{N j}+w_{N j+1}\right)^{2} \leq 8 \sum_{i=1}^{N}(N+1)\left(w_{i N}\right)^{2}+8 \sum_{j=1}^{N}(N+1)\left(w_{N j}\right)^{2} .
\end{aligned}
$$

From (A.17)-(A.18) we easily deduce (A.16). Now we estimate the right hand side in (A.16). Concerning the first term we have

$$
\sum_{i, j=0}^{N} R_{i j}^{2} \leq \frac{32}{h^{2}} \sum_{i, j=0}^{N}\left[\left(w_{i+1 j}-w_{i-1 j}\right)^{2}+\left(w_{i j+1}-w_{i j-1}\right)^{2}\right],
$$


where we have used Young's inequality and the fact that $i, j \leq h^{-1}$. In (A.19), the first term is estimated as follows

$$
\begin{aligned}
& \sum_{i, j=0}^{N}\left(w_{i+1 j}-w_{i-1 j}\right)^{2} \\
& \quad=\frac{1}{2} \sum_{i, j=0}^{N}\left[\left(w_{i+1 j}-w_{i j}+w_{i j}-w_{i-1 j}\right)^{2}+\left(w_{i+1 j}-w_{i j+1}+w_{i j+1}-w_{i-1 j}\right)^{2}\right] \\
& \quad \leq \sum_{i, j=0}^{N}\left[\left(w_{i+1 j}-w_{i j}\right)^{2}+\left(w_{i j}-w_{i-1 j}\right)^{2}\right]+\sum_{i, j=1}^{N}\left[\left(w_{i+1 j}-w_{i j+1}\right)^{2}+\left(w_{i j+1}-w_{i-1 j}\right)^{2}\right] \\
& \quad=\sum_{i, j=0}^{N}\left[2\left(w_{i+1 j}-w_{i j}\right)^{2}+\left(w_{i+1 j}-w_{i j+1}\right)^{2}+\left(w_{i+1 j+1}-w_{i j}\right)^{2}\right]-2 \sum_{j=0}^{N}\left(w_{N j}\right)^{2},
\end{aligned}
$$

and an analogous formula holds for the second term in (A.19).

Substituting this simplification of (A.19) into (A.16) we easily obtain

$$
\begin{aligned}
& \sum_{i, j=0}^{N}\left(a_{i i+1}^{j j+1}+R_{i j}\right)^{2} \\
& \quad \leq \frac{64}{h^{2}} \sum_{i, j=0}^{N}\left[\left(u_{i+1 j}-w_{i j}\right)^{2}+\left(u_{i j+1}-u_{i j}\right)^{2}+\left(w_{i+1 j}-w_{i j+1}\right)^{2}+\left(w_{i+1 j+1}-w_{i j}\right)^{2}\right],
\end{aligned}
$$

which allows us to estimate (A.15). In fact, by Young's inequality we obtain

$$
\begin{aligned}
& \sum_{i, j=0}^{N} a_{i i+1}^{j j+1} b_{i i+1}^{j j+1}+C_{2} \leq \frac{8}{h}\left(\sum_{i, j=0}^{N}\left(b_{i i+1}^{j j+1}\right)^{2}\right)^{1 / 2} \\
& \quad \times\left(\sum_{i, j=0}^{N}\left[\left(w_{i+1 j}-w_{i j}\right)^{2}+\left(w_{i j+1}-w_{i j}\right)^{2}+\left(w_{i+1 j}-w_{i j+1}\right)^{2}+\left(w_{i+1 j+1}-w_{i j}\right)^{2}\right]\right)^{1 / 2} \\
& \leq 16 \sqrt{3}\left[\sum_{i, j=0}^{N}\left(\frac{b_{i i+1}^{j j+1}}{4}\right)^{2}+\frac{1}{3} \sum_{i, j=0}^{N}\left[\left(w_{i+1 j}-w_{i j}\right)^{2}+\left(w_{i j+1}-w_{i j}\right)^{2}\right.\right. \\
& \left.\left.\quad+\left(w_{i+1 j}-w_{i j+1}\right)^{2}+\left(w_{i+1 j+1}-w_{i j}\right)^{2}\right]\right]=32 \sqrt{3} E_{h}(t) .
\end{aligned}
$$

Therefore,

$$
\left[\sum_{i, j=0}^{N} a_{i i+1}^{j j+1} b_{i i+1}^{j j+1}+C_{2}\right]_{0}^{T} \leq 32 \sqrt{3}\left(E_{h}(T)+E_{h}(0)\right) \leq 64 \sqrt{3} E_{h}(0) .
$$

This concludes the proof of Lemma A.2.

Acknowledgements. The authors are grateful to Professor E. Zuazua for several suggestions and remarks related with this work. 


\section{References}

Banks H. T., Ito K. and Wang C., Exponentially stable approximations of weally damped wave equations. Estimation and control of distributed parameter systems (Vorau, 1990), 1-33, Internat. Ser. Numer. Math., 100, Birkhäuser, Basel, 1991.

Bensoussan A., On the general theory of exact controllability for shew symmetric operators, Acta Appl. Math., 20(3) (1990),197-229.

Cohen G. C., Higher-order Numerical Methods for Transient Wave Equations, Scientific Computation, Springer Verlag, Berlin, 2002.

Glowinski R., Ensuring well-posedness by analogy; Stokes problem and boundary control for the wave equation, J. Comput. Phys., 103, 189-221 (1991).

Glowinski R., Kinton W. and Wheeler M. F., A mixed finite element formulation for the boundary controllability of the wave equation, Int. J. Numer. Methods Eng. 27(3), 623-636 $(1989)$.

Glowinski R., Li C. H. and Lions J.-L., A numerical approach to the exact boundary controllability of the wave equation (I). Dirichlet controls: Description of the numerical methods, Jap. J. Appl. Math. 7 (1990), 1-76.

Glowinski R. and Lions J.-L., Exact and approximate controllability for distributed parameter systems, Acta Numerica 1996, 159-333.

Infante J. A. and Zuazua. E., Boundary observability for the space semi-discretization of the 1-D wave equation, M2AN, 33(2) (1999), 407-438.

Kappel F. and Ito K., The Trotter-Kato theorem and approximations of PDE's, Math. of Comput., 67 (1998), 21-44.

Lions J.-L., ContrôlabilitÉ EXACTE PERTURBations ET STABILISATION DE SYSTÈmES Distribués, Tome 1, Masson, Paris, 1988.

Münch A., Family of implicit schemes uniformly controllable for the 1-D wave equation, C.R. Acad. Sci. Paris, Série I, 339, (2004) 733-738. 
Roberts J. E. and Thomas J.-M., MiXed AND hYBRID METHODS, in Handbook of Numerical Analysis, vol. 2, P. G. Ciarlet and J.-L. Lions eds., North-Holland, Amsterdam, The Netherlands, 1989.

Raviart P. A. and Thomas J.-M., INTRODUCTION À L'ANALYSE NUMÉRIQUE deS ÉQUATIONS AuX DÉRIVÉES PARTIElles, Masson, Paris, 1983.

Trefethen L. N., Group Velocity in Finite Difference Schemes, SIAM Rev., 24 (1982), 113136.

Zuazua E., Boundary observability for the finite difference space semi-discretizations of the 2-D wave equation in the square, J. Math. Pures Appl., 78 (1999), 523-563.

Zuazua E., Propagation, Observation. Control and Numerical Approximation of Waves, SIAM Review, 47(2), (2005), 197-243. 